Portland State University

PDXScholar

Electrical and Computer Engineering Faculty

Publications and Presentations

$10-2016$

\title{
Memristor-Based Volistor Gates Compute Logic with Low Power Consumption
}

\author{
Muayad Aljafar \\ Portland State University \\ Marek Perkowski \\ Portland State University, marek.perkowski@pdx.edu
}

Follow this and additional works at: https://pdxscholar.library.pdx.edu/ece_fac

Part of the Electrical and Computer Engineering Commons

Let us know how access to this document benefits you.

\section{Citation Details}

Published as, Aljafar, M., Long, P., \& Perkowski, M. (2016). Memristor-Based Volistor Gates Compute Logic with Low Power Consumption. BioNanoScience, 6(3), 214-234.

This Post-Print is brought to you for free and open access. It has been accepted for inclusion in Electrical and Computer Engineering Faculty Publications and Presentations by an authorized administrator of PDXScholar. Please contact us if we can make this document more accessible: pdxscholar@pdx.edu. 


\title{
Memristor-Based Volistor Gates Compute Logic With Low Power Consumption
}

\author{
Muayad Aljafar, Paul Long, and Marek Perkowski,
}

M. Aljafar, P. Long. and M. Perkowski are with the Department of Electrical and Computer Engineering, Portland State University, Portland, OR 97201, USA (email: muayad@pdx.edu; pwl@pdx.edu; mperkows@ee.pdx.edu).

Abstract - We introduce a novel volistor logic gate which uses voltage as input and resistance as output. Volistors rely on the diode-like behavior of rectifying memristors. We show how to realize the first logic level, counted from the input, of any Boolean function with volistor gates in a memristive crossbar network. Unlike stateful logic, there is no need to store the inputs as resistances, and computation is performed directly. The fan-in and fan-out of volistor gates are large and different from traditional memristor circuits. Compared to solely memristive stateful logic, a combination of volistors and stateful inhibition gates can significantly reduce the number of operations required to calculate arbitrary multi-output Boolean functions. The power consumption of volistor logic is computed and compared with the power consumption of stateful logic using the simulation results obtained by LTSpice-when implemented in a $1 \times 8$ or an $8 \times 1$ crosspoint array, volistors consume significantly less power.

Index Terms - Crosspoint array, logic computation, memristive crossbar, rectifying memristor, stateful inhibition, volistor logic.

\section{INTRODUCTION}

Stateful logic computation with memristors is an area of active research. Borghetti et al. [1] proposed realizing stateful logic via material implication (IMP). In classical stateful memristive circuits, logic signals utilize resistances on inputs and outputs, meaning the previous resistance state of the memristor affects the operations. In contrast, volistors do not use the previous resistance state in calculations. Other stateful logic gates have been proposed as well, e.g. inhibition (INH) [2] or AND [3]. INH gate implements Boolean function $a \bar{b}$ where $a$ and $b$ are positive and negative inputs, respectively. In stateful logic, the logic values are encoded by the resistance states of memristors. Stateful logic is usually performed in a generic structure called a crossbar array. Leakage pathways due to the half-select of memristors in a crossbar row or column can be suppressed by using rectifying memristors [4]. One key disadvantage of stateful logic is that a long sequence of operations is required to implement an arbitrary Boolean function. 
Memristor ratioed logic (MRL) [5] is another approach to logic computation and is based on the resistive ratio of nonrectifying memristors. In MRL, logic values are voltage-based pulses and circuit structures are dependent entirely on the Boolean function being implemented. The stable output voltage depends only on input voltages regardless of the resistance of the memristors; however, the resistances affect the propagation delay of the gate. MRL gates consume both dynamic and static power. Further, as it lacks the inversion function, MRL is not logically complete without CMOS inverters.

In this paper we introduce a new concept in memristor logic. We call it the volistor gate (voltage-resistor gate) which has voltage-based inputs and resistance based-outputs. Therefore, volistor gates can only be used in the first level of logic implementation. This level can be composed of various types of gates and be complex. Logic synthesis methods with volistors are therefore different from classical logic synthesis. Volistors are implemented in generic crossbar arrays of rectifying memristors [6]. Unlike stateful logic, voltage pulses are the actual inputs to the volistor gates. It is always assumed that complemented input variables are available as voltage signals at no additional cost. Sometimes it is required that the logic input signals are available in both positive and negative forms, e.g. $x$ and $\bar{x}$. In addition, there is no propagation penalty as it exists in MRL. In a $1 \times 8$ crosspoint array, the propagation delay in volistors is shorter than in stateful INH. In a $1 \times 8$ crosspoint array, multi-input multi-output volistor gates consume less power than the corresponding stateful logic. The outputs of volistors are stored as the resistances of the target memristors. To provide the correct functionality, target memristors need to be initially closed, i.e. target memristors must be set to the low resistance state. With different coding schemes, either a volistor OR and NAND logic set or a volistor NOR and AND logic set can be realized in the same crossbar array. For instance, if the closed state of a memristor encodes logic ' 0 ' and the open state of a memristor encodes logic ' 1 ', the set of OR and NAND operations is implemented. The reverse encoding scheme implements the NOR and AND logic set. With volistor logic, crossbar drivers need to supply only three voltage levels: $\mathrm{V}^{+}, \mathrm{V}^{-}$, or $0 \mathrm{~V}$. In addition, the control circuitry must be capable of setting arbitrary nanowires in the crossbar to either high impedance (HZ) or grounding these nanowires through load resistors $\mathrm{R}_{\mathrm{G}}$. Obviously, the volistor network layer cannot implement every Boolean function such as Sum of Products. However, a combination of a volistor NOR and AND with stateful INH, or their dual logic of volistor OR and NAND with stateful IMP, can both be used to realize arbitrary multi-output Boolean functions. As will be presented, this hybrid realization is faster than an equivalent circuit realized with only stateful gates. The speed comparison of hybrid and stateful logic circuits will be presented in Section 4. 
In Section 2, the hysteresis behavior of rectifying memristors is reviewed. In Section 3, volistor gates are described. In Section 4, the synthesis of arbitrary Boolean functions is discussed. In Section 5, the power consumption of volistor gates is computed and compared with stateful logic. In section 6, volistors in memory application are discussed. Section 7 is a summary of the work.

\section{RECTIFYING MEMRISTORS}

We used a rectifying memristor [4], [6] as a linear bistable device [7]. The behavior of a rectifying memristor is defined in (1), following [7]. Equation (1) describes a simplified model of diode-like memristor $M$ demonstrated practically in [4]. $R_{M}$ denotes resistance of memristor $M ; s$ is the state variable of $M$ normalized to a real number in the range 0 through $1, s \in[0,1]$, and therefore $R_{C L O S E D} \leq R_{M} \leq R_{O P E N} ; v$ is the voltage applied across $M$.

$R_{M}= \begin{cases}R_{O P E N}\left(\frac{R_{C L O S E D}}{R_{O P E N}}\right)^{s} & v \geq 0 \\ R_{O P E N} & v<0\end{cases}$

$R_{O P E N}$ denotes high resistance state of memristor $M ; R_{C L O S E D}$ is low resistance state of memristor $M$. It is assumed that $R_{O P E N}=500 \mathrm{M} \Omega$ and $R_{C L O S E D}=500 \mathrm{~K} \Omega$ which are consistent with empirical results reported in [4]. The dynamic behavior of the state variable $s$ is such that $s$ changes in time as described in the linear differential Equation (2). In Equation (2), $v_{C L O S E}$ is a positive threshold voltage; $v_{O P E N}$ is a negative threshold voltage. For simplicity, it is assumed that the switching threshold voltages $V_{C L O S E}$ and $V_{O P E N}$ are symmetric where $V_{C L O S E}=-V_{O P E N}=1 \mathrm{~V} ; \alpha$ is a positive constant associated with the switching rate of the memristor. Here, $\alpha$ is assumed to be $125 \times 10^{7}(\mathrm{~V} . \mathrm{s})^{-1}$, following [7].

$\frac{d s}{d t}=\left\{\begin{array}{cc}\alpha\left(v-v_{C L O S E}\right) & v>v_{C L O S E} \\ \alpha\left(v-v_{O P E N}\right) & v<v_{O P E N} \\ 0 & \text { elsewhere }\end{array}\right.$

Fig. 1a shows the $i-v$ characteristic of rectifying memristor $M$ described in (1) and (2). In this work, the positive programming voltage is defined $v=1.2 \mathrm{~V}$ and denoted by $\mathrm{V}_{\mathrm{SET}}$; the application of $\mathrm{V}_{\mathrm{SET}}$ will close switch $M$ when it is open. Also, the negative programming voltage is defined $v=-1.2 \mathrm{~V}$ and denoted by $\mathrm{V}_{\mathrm{CLEAR}}$; the application of $\mathrm{V}_{\text {CLEAR }}$ will open switch $M$ when it is closed. Substituting all related values in Equation (2) results in: 


$$
\frac{d s}{d t}=\left\{\begin{array}{cc}
0.2 \alpha & v=1.2 \\
-0.2 \alpha & v=-1.2 \\
0 & \text { elsewhere }
\end{array}\right.
$$

The straightforward analytical solution of this differential equation is

$$
s(t+1)= \begin{cases}0.2 \alpha T^{+} & v=1.2 \\ -0.2 \alpha T^{-} & v=-1.2 \\ s(t) & \text { elsewhere }\end{cases}
$$

Since $s$ is normalized to interval [0,1], for the given values of $\alpha$ and $v$, the assumption of $s(t+1)=1$ results in the switching delay of $T^{+}=4 n s$. The desired solution for (2) is

$$
s(t+1)=\left\{\begin{array}{ccr}
1 & v & =1.2 \\
0 & v & =-1.2 \\
s(t) & -1 & \leq v \leq 1
\end{array}\right.
$$

Note that $s(t)$ and $s(t+1)$ denote the current state and the next state of memristor $M$, respectively; for $-1 \leq v \leq$ 1 , the resistance state of memristor remains unchanged, i.e. $s(t+1)=s(t)$. Obviously, a larger $\mathrm{V}_{\mathrm{SET}}$ results in a smaller switching delay $T^{+}$, as suggested by Equation (2). Note that $s(t)$ and $s(t+1)$ denote the current state and the next state of memristor $M$, respectively; for $-1 \leq v \leq 1$, the resistance state of memristor remains unchanged, i.e. $s(t+1)=s(t)$. Obviously, a larger $\mathrm{V}_{\mathrm{SET}}$ results in a smaller switching delay $T^{+}$, as suggested by Equation (2).

This simplified model does not accurately describe the behavior of rectifying memristor $M$, for instance, the threshold voltages, $v_{C L O S E}$ and $v_{O P E N}$, are assumed to be constant, and/or the programming rate $\frac{d s}{d t}$ and the applied voltage $v$ are assumed piecewise linearly related. The experimental results of a $16 \times 16$ crossbar array fabricated in HRL Laboratories and reported in $\underline{[4]}$ are shown in Fig $1 \mathrm{~b}$ and Fig 1c. The $i-v$ characteristics of ten rectifying memristors of the crossbar array is shown in Fig. 1b. Every memristor has a different switching threshold $v_{C L O S E}$; these thresholds result in different switching rates for every memristor. Since these differences among voltage thresholds are not significant, the switching rates of memristors vary only slightly. A slight increase in the pulse width driving the crossbar array ensures a complete switching of every target memristor in the crossbar array during logic operations. Fig. 1c shows the threshold voltage distribution of all memristors in the $16 \times 16$ crossbar array. This threshold voltage varies in the range of $2.1 \mathrm{~V}-2.5 \mathrm{~V}$. Also, Fig. $1 \mathrm{~b}$ shows a nearly piecewise linear relations between $i$ and $v$ in interval $\left[0, v_{C L O S E}\right]$.

In this work, the exact behavior of rectifying memristor for $v \epsilon\left(0, v_{C L O S E}\right)$ is not required. What affects the results of our work are the $\frac{\mathrm{R}_{\mathrm{OPEN}}}{\mathrm{R}_{\text {CLOSED }}}$ ratio, the $\frac{\mathrm{I}^{-}}{\mathrm{I}^{+}}$ratio where $\mathrm{I}^{-}$is reverse biased current and $\mathrm{I}^{+}$is forward biased current, and 

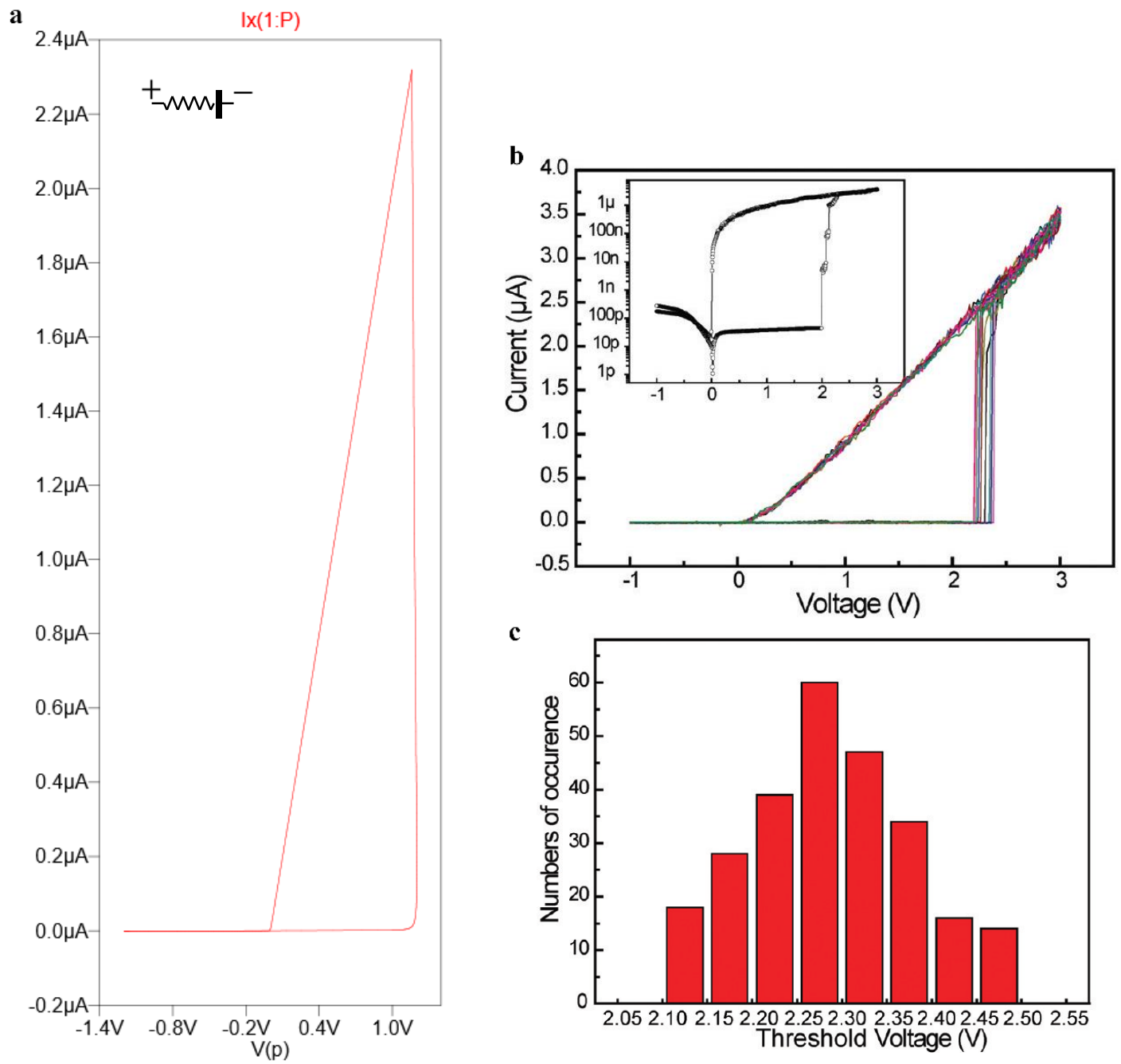

Fig. 1 (a) The $\mathrm{i}-\mathrm{v}$ characteristic of rectifying memristor $\mathrm{M}$ described in (1) and (2). A sinusoidal input voltage with frequency $25 \mathrm{MHz}$ and amplitude $1.2 \mathrm{~V}$ is applied to memristor $\mathrm{M}$. The inset shows the symbolic diagram of a rectifying memristor. (b) The $\mathrm{i}-\mathrm{v}$ characteristics of 10 different rectifying memristors in the crossbar array. The inset shows the $\mathrm{i}-\mathrm{v}$ characteristics plotted in log scale demonstrating current suppression at negative bias in the on-state [4]. (c) Threshold voltage distribution of 256 cells in the fabricated crossbar array. The threshold voltage is defined as the voltage at which the measured current is above $10^{-6} \mathrm{~A}[4]$.

the switching rate of memristor. In our used model the resistance ratio is $10^{3}$ which is consistent with the empirical results reported in $\left[4, \underline{6]}\right.$, i.e. the resistance ratio is from three to six orders of magnitude $\left(10^{3}-10^{6}\right)$.

The current ratio in the model that we used [7] is $10^{-3}$ which is much larger that the empirical results reported in [6], i.e. $\mathrm{I}^{-}<10^{-13} \mathrm{~A}$ and $\frac{\mathrm{I}^{-}}{\mathrm{I}^{+}}<10^{-6}$. Thus, a more precise memristor model would show even less power consumption in volistors. The switching rate of memristor is in accordance with empirical results reported in [8-9]. As will be elaborately discussed in Section 3, due to the circuit architecture, the large resistance ratio, and the small current ratio, memristors are only operated in range $v \leq 0$. Thus the simplified model for rectifying memristors can 
be sufficient to compute both delay and power consumed in volistors.

\section{VOLISTOR LOGIC}

In this section volistor logic is introduced. The key idea behind volistor logic is that inputs are voltages and outputs are resistances. This is a significant change from the way the voltage drivers are used in stateful logic; however, the target memristor is still used as a memory element where the output is stored. The basic volistor logic gates are: inverter, $k$-input NOR and $k$-input AND, and their duals: $k$-input OR and $k$-input NAND. In the following subsections we define the basic architecture required for logic computations and describe the basic logic gates.

\subsection{Crosspoint architecture}

The basic circuit structures for volistor logic computations are the $1 \times 2$ crosspoint array and $2 \times 1$ crosspoint array depicted in Fig. 2. The crosspoint array is a horizontal or a vertical vector of memristors, i.e. a one-dimensional array. Fig. 2a shows a generic structure for logic operations. The circuit is comprised of two rectifying memristors, labeled $\mathrm{S}$ and T, electrically connected through the common horizontal nanowire HL. Just as in stateful IMP, S denotes the source memristor and T the target memristor. The vertical nanowire VL1 connected to source memristor S conveys input signal $\mathrm{V}_{\text {in }}$. Let $\mathrm{V}^{+}=0.6 \mathrm{~V}$ and $\mathrm{V}^{-}=-0.6 \mathrm{~V}$. The logical coding scheme for $\mathrm{V}_{\text {in }}$ is defined as follows: $\mathrm{V}^{+}$encodes logic ' 1 ', denoted $\operatorname{vin}=1 ; 0$ Volts encodes logic ' 0 ', denoted $\operatorname{vin}=0$. The vertical nanowire VL2 connected to target memristor $\mathrm{T}$ carries a bias voltage, $\mathrm{V}_{\text {bias }}=\mathrm{V}^{-}$. Memristor $\mathrm{T}$ acts as a switch whose resistivity state $t$ represents the output of the crosspoint array. When $\mathrm{T}$ is open, its high-resistivity state encodes logic ' 0 ', i.e. $t=0$. When $\mathrm{T}$ is closed, its low- resistivity state encodes logic ' 1 ', $t=1$. This interpretation of the resistivity state of $\mathrm{T}$ is used for performing NOR/AND logic set; another interpretation will be discussed in Section 3.5. Prior to logic computation, both memristors $\mathrm{S}$ and $\mathrm{T}$ must be closed. To unconditionally close a memristor, it must be forward biased by $\mathrm{V}_{\mathrm{SET}}$. Unlike the target memristor, the source memristor $\mathrm{S}$ acts as a diode. The $1 \times 2$ crosspoint array must satisfy (3).

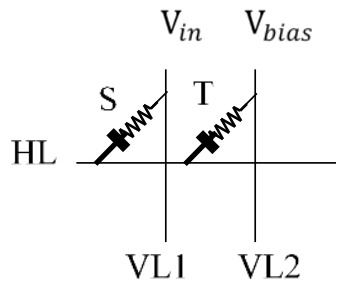

(a)

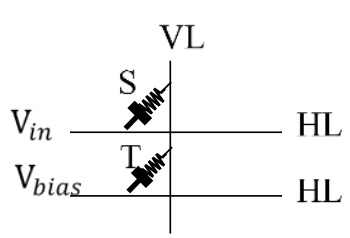

(b)

Fig. 2 (a) A $1 \times 2$ crosspoint array; (b) a $2 \times 1$ crosspoint array 


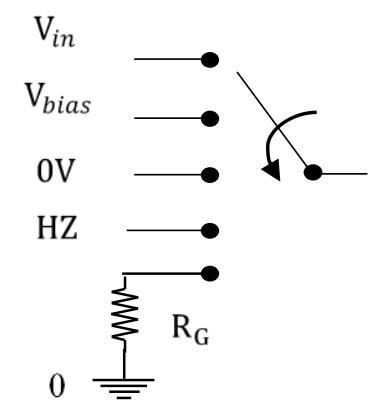

Fig. 3 The symbolic illustration of driver circuitry connected to each nanowire

$\left\{\begin{array}{l}V_{O P E N}<V_{\text {bias }}<0 V \\ V_{\text {bias }}-V_{\text {in }}=V_{C L E A R}\end{array}\right.$

Each nanowire must be either driven by one of the voltages $\mathrm{V}_{\text {in }}, \mathrm{V}_{\text {bias }}$, and $\mathrm{OV}$ or terminated with high impedance $\mathrm{HZ}$ or grounded by load resistor $\mathrm{R}_{\mathrm{G}}$ as shown in Fig. 3. All these connections are realized with CMOS switches shown symbolically in Fig. 3. $R_{G}$ is defined as geometric mean of $R_{\text {OPEN }}$ and $R_{C L O S E D}, \sqrt{R_{O P E N} \cdot R_{\text {CLOSED }}}=15 M \Omega$. The crosspoint array operates by the simultaneous application of $\mathrm{V}_{\text {in }}$ and $\mathrm{V}_{\text {bias }}$ to $\mathrm{S}$ and $\mathrm{T}$, respectively. Since $\mathrm{V}_{\text {in }}>\mathrm{V}_{\text {bias }}$, Ohm's Law requires a flow of current through the array. However, given the structure of the array, the application of these voltages forward biases $\mathrm{S}$ and reverse biases $\mathrm{T}$ thus suppressing the flow of current. This means that $\mathrm{V}_{\mathrm{HL}}$ $\approx \mathrm{V}_{\text {in }}$ where $\mathrm{V}_{\mathrm{HL}}$ is the voltage on HL. If (3) is satisfied, the voltage across $\mathrm{T}$ will toggle that memristor, i.e. the new state of target memristor becomes $t=0$.

$\left\{0 V<V_{\text {bias }}<V_{\text {CLOSED }}\right.$

$\left\{V_{\text {in }}-V_{\text {bias }}=V_{\text {CLEAR }}\right.$

The crosspoint arrays shown in Fig. 2 can be scaled to $1 \times n$ and $n \times 1$ arrays, allowing for multi-input multi-output volistor logic functions. The $1 \times n$ and $n \times 1$ crosspoint arrays must satisfy (3) and (4), respectively. In these arrays, logic computation is achieved by implementing the wired $\mathrm{OR}$ function, i.e. $\mathrm{V}_{\mathrm{HL}}$ or $\mathrm{V}_{\mathrm{VL}}$ denotes the logical $\mathrm{OR}$ of inputs $\operatorname{vin}_{1}, \ldots, \operatorname{vin}_{k}$ where $\mathrm{V}_{\mathrm{VL}}$ is the voltage on vertical nanowire $\mathrm{VL}$ and $1 \leq k<n$.

\subsection{Volistor NOT gate in a crosspoint array}

An inverter is the simplest gate to realize in volistor logic; the symbolic diagram is shown in Fig. 4a. Take, for example, the case where $(\operatorname{vin}, t)=(1,1)$ i.e. $\operatorname{vin}=1$ and $t=1$. Both source and target memristors are initially closed. Single-output volistor NOT can be realized in either a $1 \times 2$ or a $2 \times 1$ crosspoint array. When realized in a $1 \times 2$ array, $\mathrm{V}_{\text {bias }}$ must be a negative voltage, $\mathrm{V}_{\text {in }}$ must be greater than or equal to $0 \mathrm{~V}$, per (3), and horizontal nanowire HL must 
be connected to a high impedance, HZ. Connecting HL to HZ allows $\mathrm{V}_{\text {in }}$ to manifest on HL. Based on Ohm's Law, current should flow through the array, since $\mathrm{V}_{\text {in }}-\mathrm{V}_{\text {bias }}>0 \mathrm{~V}$. However, memristor $\mathrm{T}$ is reverse biased and thus

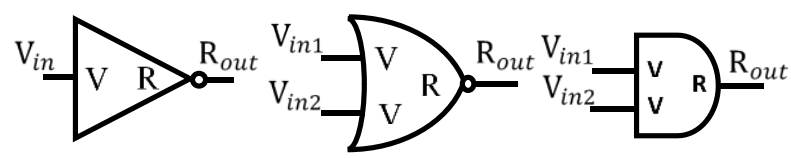

(a)

(b)

(c)

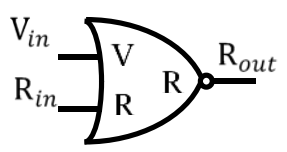

(d)

Fig. 4 Symbolic notation for volistor single-input and two-input logic gates. (a) Volistor inverter; (b) two-input volistor NOR gate; (c) two-input volistor AND gate; (d) mixed-input NOR gate. Inside the gates, symbols V and R denote whether a signal is a voltagebased or resistance-based respectively

suppresses the flow of current. In this case, the voltage drop across memristor $\mathrm{S}$ is $1.198 \mathrm{mV}$, i.e. the voltage on HL equals $598.801 \mathrm{mV}$. The voltage drop across $\mathrm{T}$ is $1.198 \mathrm{~V}$ which is sufficient to open memristor T. This is the desired behavior of the inverter function, that it results in $($ vin, $t)=(1,0)$. Given the parameters introduced in Section 2 , the propagation delay of single-output volistor NOT is $4.044 \mathrm{~ns}$ as shown in Fig. $5 \mathrm{~b}$.

Single-output volistor NOT can be extended to an arbitrary fan-out which corresponds to a multi-output gate realized in arrays of types $1 \times n$ or $n \times 1$. The multi-output NOT gate stores $\overline{v \imath n}$ in up to $n-1$ target memristors in any arbitrary location in the array. The role of each memristor is determined by its driver, i.e. source memristors are driven by $\mathrm{V}_{\text {in }}$ and target memristors by $\mathrm{V}_{\text {bias }}$. Interestingly, this means that a memristor's function is independent of its position in the crosspoint array. Fig. 5a shows a four-output volistor NOT gate implemented in a $1 \times 8$ array. Source memristor S is driven by $\mathrm{V}_{\text {in }}$ and target memristors $\mathrm{T}_{1} \cdots \mathrm{T}_{4}$ are driven by $\mathrm{V}_{\text {bias }}$. Memristors $\mathrm{X}_{1}, \mathrm{X}_{2}$ and $\mathrm{X}_{3}$ are terminated to $\mathrm{HZ}$ and do not take part in the circuit's operation. The proper operation of a 63-output NOT gate with vin $=1$ implemented in a $1 \times 64$ array and simulated in LTSpice is depicted in Fig. 5c. $\mathrm{V}_{\mathrm{HL}}$ is $528.881 \mathrm{mV}$ and all 63 target memristors are switched off successfully in 6.223 ns. Fig. $5 \mathrm{~d}$ shows the desired behavior of volistor NOT implemented in a $1 \times 2$ array when vin $=0 . \mathrm{V}_{\mathrm{HL}}$ is $-599.4 \mu \mathrm{V}$ and the target switch remains closed. Fig. 5e shows the behavior of a 63-output volistor NOT implemented in a $1 \times 64$ array where $\operatorname{vin}=0$. This results in $\mathrm{V}_{\mathrm{HL}}=-35.559 \mathrm{mV}$ and all target switches remaining closed. 
Table 1 summarizes these configurations and their effects on $\mathrm{V}_{\mathrm{HL}}$. A NOT with an arbitrary fan-out can be realized in one pulse. We discuss this gate for completeness; in practice, it is not required since the logical negation can be created by appropriately selecting the vin values. In this paper all volistor gates can appear only at the input layer.

\subsection{Volistor NOR gate in a crosspoint array}

The second basic gate in volistor logic is NOR. Fig. 4b shows the symbolic diagram of a two-input volistor NOR gate. Since $1 \times n$ arrays have been previously discussed, in this subsection $n \times 1$ arrays are considered. Take, for example, a two-input NOR gate where $\left(\operatorname{vin}_{1}, \operatorname{vin}_{2}, t\right)=(1,0,1)$. This requires the application of input data to $S_{1}$ and $S_{2}$ and $\mathrm{V}_{\text {bias }}$ to $\mathrm{T}$, as shown in Fig. 6. Since the function is realized in a $3 \times 1$ crosspoint array, equation (4) requires $\mathrm{V}_{\text {bias }}>0 \mathrm{~V}$ and $\mathrm{V}_{\text {in }} \leq 0 \mathrm{~V}$. Based on Ohm's Law, since $\mathrm{V}_{\text {bias }}-\mathrm{V}_{\text {in }}>0$, a current should flow through the crosspoint array.

Table 1 Implementation of multi-output volistor not gate

\begin{tabular}{|c|c|c|c|c|c|}
\hline $\begin{array}{c}\text { Crosspoint } \\
\text { Array }\end{array}$ & vin & $\begin{array}{c}\text { Number of } \\
\text { Outputs }\end{array}$ & $\begin{array}{c}\mathrm{V}_{\mathrm{HL}} \\
(\mathrm{mV})\end{array}$ & $\begin{array}{c}\mathrm{t}_{\mathrm{d}} \\
(\mathrm{ns})\end{array}$ & Fig. \\
\hline $1 \times 2$ & 1 & 1 & 598.801 & 4.065 & Fig. 5b \\
\hline $1 \times 64$ & 1 & 63 & 528.881 & 6.223 & Fig. 5c \\
\hline $1 \times 2$ & 0 & 1 & -0.599 & $\begin{array}{c}\text { Not } \\
\text { applicable }\end{array}$ & Fig. 5d \\
\hline $1 \times 64$ & 0 & 63 & -35.559 & $\begin{array}{c}\text { Not } \\
\text { applicable }\end{array}$ & Fig. 5e \\
\hline
\end{tabular}


However, $S_{2}$ and $\mathrm{T}$ are both reverse biased and thus they suppress the flow of current. The voltage drop across $\mathrm{S}_{1}$ is $1.796 \mathrm{mV}$, i.e. $\mathrm{V}_{\mathrm{VL}}=-598.203 \mathrm{mV}$. The voltage drop across $\mathrm{T}$ is $-1.198 \mathrm{~V}$ which is sufficient to open $\mathrm{T}$. Recall that all memristors are initially closed. The gate is realized by implementing wired-OR, i.e. the voltage on VL is the logical OR of $\operatorname{vin}_{1}$ and $\operatorname{vin}_{2}$. As before, $\mathrm{V}_{\text {in }}$ causes memristors $\mathrm{S}_{1}$ and $\mathrm{S}_{2}$ to behave as diodes and $\mathrm{V}_{\text {bias }}$ causes memristor $\mathrm{T}$ to behave as a switch. The output of the gate is the new state of the target switch T, either open or closed. Table 2 shows $\mathrm{V}_{\mathrm{VL}}$ and $t_{\mathrm{d}}$ for various combinations of input values of a two-input, single-output volistor NOR gate implemented in a $3 \times 1$, and a sixty three-input, single-output volistor NOR gate implemented in a $64 \times 1$ crosspoint array. Note that with

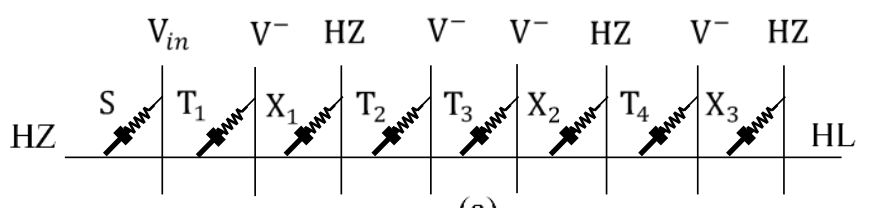

(a)

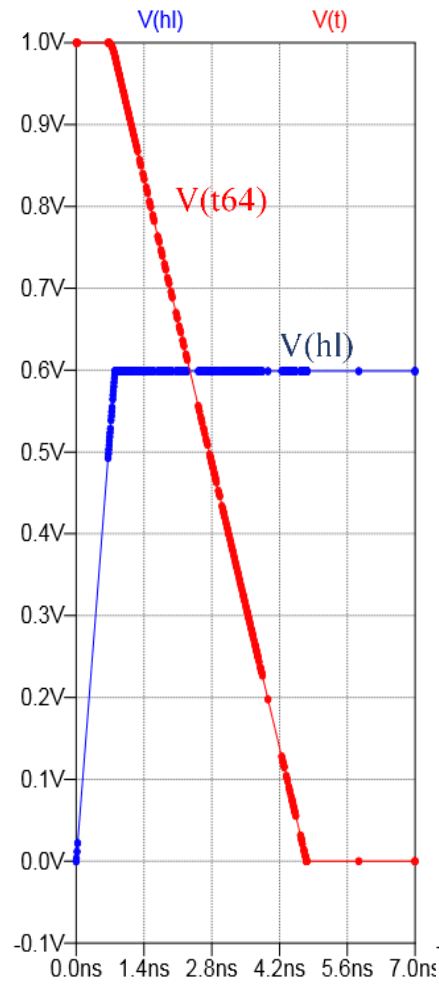

(b)
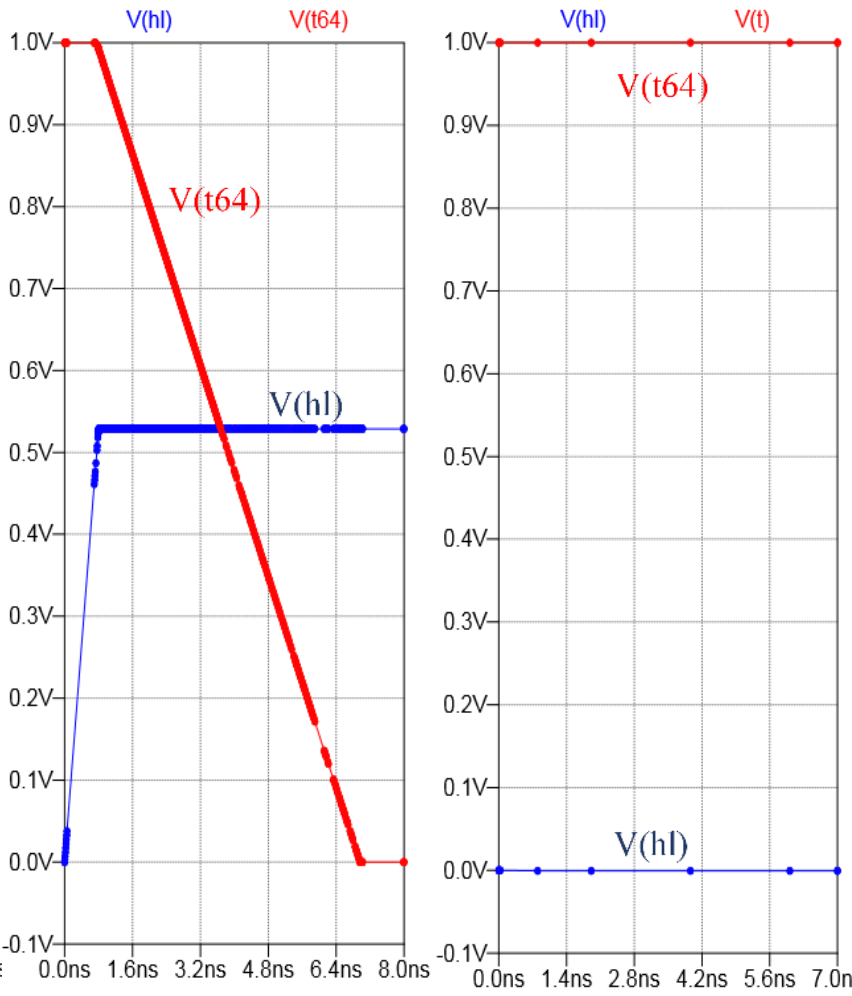

Time (ns)

(c) (d)

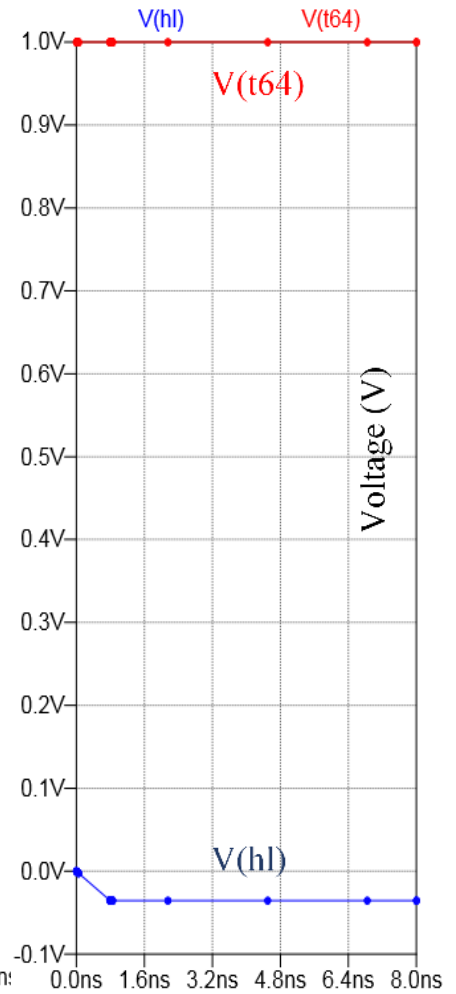

(e)

Fig. 5 Volistor NOT behavior. (a) A $1 \times 8$ crossbar array implementing a four-output NOT and showing arbitrary nature of the locations of S and T. The contribution of each memristor is determined by the voltage driver to which it is connected. The horizontal nanowire is connected to $\mathrm{HZ}$. (b) The operation of a one output NOT in a $1 \times 2$ array. $V(\mathrm{hl})$ stabilizes at $\approx 600 \mathrm{mV}$ indicating vin= ' 1 ' manifesting on $\mathrm{HL}$. In addition $\mathrm{V}(\mathrm{t})$, which is the SPICE model's representation of $t$, toggles to $0 \mathrm{~V}\left(t={ }^{\prime} 0\right.$ '). (c) The operation of a 63 output NOT in a $1 \times 64$ array. $V(\mathrm{hl})$ stabilizes and $\mathrm{V}(\mathrm{t})$ toggles as in (b). (d) The operation of a one output NOT in a $1 \times 2$ array. $\mathrm{V}(\mathrm{hl})$ stabilizes at $\approx-600 \mu \mathrm{V}$ indicating vin='0' manifesting on $\mathrm{HL}$. As a result, $\mathrm{V}(\mathrm{t})$ 
Table 2 Implementation of multi-in single-out volistor nor gate

\begin{tabular}{|c|c|c|c|c|}
\hline $\begin{array}{c}\text { Crosspoint } \\
\text { Array }\end{array}$ & \multicolumn{2}{|c|}{ Number of Inputs } & $\mathrm{V}_{\mathrm{VL}}$ & $\mathrm{t}_{\mathrm{d}}$ \\
\cline { 2 - 3 }$(\mathrm{ns})$ & vin= 0 & vin=1 & $(\mathrm{mV})$ & $(\mathrm{ns})$ \\
\hline \multirow{3}{*}{$2 \times 1$} & 0 & 2 & -599.400 & 4.048 \\
\cline { 2 - 5 } & 1 & 1 & -598.203 & 4.068 \\
\cline { 2 - 5 } & 2 & 0 & 0.300 & Not applicable \\
\hline \multirow{3}{*}{$64 \times 1$} & 63 & 0 & 0.010 & Not applicable \\
\cline { 2 - 5 } & 0 & 63 & -599.981 & 4.035 \\
\cline { 2 - 5 } & 50 & 13 & -597.609 & 4.082 \\
\hline
\end{tabular}

more logic ' 1 ' inputs the value of $\mathrm{V}_{\mathrm{VL}}$ approaches $\mathrm{V}_{\text {in }}$ and the propagation delay $\mathrm{t}_{\mathrm{d}}$ gets shorter. Volistor logic allows a multi-input NOR gate to be realized in only one pulse.

\subsection{Volistor AND gate in a crosspoint array}

The third basic gate in volistor logic is AND. This gate is realized as NOR with negated literals of the desired product applied to the crosspoint array. For example, the desired product $\bar{a} b \bar{c}$ is realized by applying $\left(\operatorname{vin}_{1}, \operatorname{vin}_{2}, \operatorname{vin}_{3}\right)=(1$, $0,1)$ to the memristors. As a result, the voltage across $\mathrm{T}$ is sufficient to toggle $t$ to ' 0 ' where $t=\overline{a+\bar{b}+c}$ and according to the De Morgan's Law $\overline{a+\bar{b}+c}=\bar{a} b \bar{c}$ which is the desired result. The AND gate can be scaled to perform multi-input multi-output operations. The details are the same as described for volistor NOR. In this work, it is assumed that negated inputs are always available at the same cost as the non-negated inputs. Volistor logic allows a multi-input AND to be realized in only one pulse.

\subsection{Volistor OR and NAND gates in a crosspoint array}

In all volistor logic gates discussed above, the logical coding scheme of memristive switch $\mathrm{T}$ is defined as follows: an open switch encodes logic ' 0 ' and a closed switch encodes logic ' 1 '. This scheme allows direct implementation of volistor NOR/AND; however, it does not allow for direct implementation of volistor OR/NAND. Therefore, executing a circuit with a volistor OR/NAND gate would require two consecutive pulses.

However, if the logical coding scheme of the memristor switch $T$ is reversed i.e. the open switch encodes logic ' 1 ' and the closed switch encodes logic ' 0 ', then the OR/NAND gate can be realized in one pulse. This scheme allows a direct realization of volistor OR in the same manner as volistor NOR described in Section 3.3. Likewise, volistor NAND is realized by applying negated input logic values to the crosspoint array in the same manner as the volistor AND described in Section 3.4. The designer could use one of the two encoding schemes for the entire system or use 


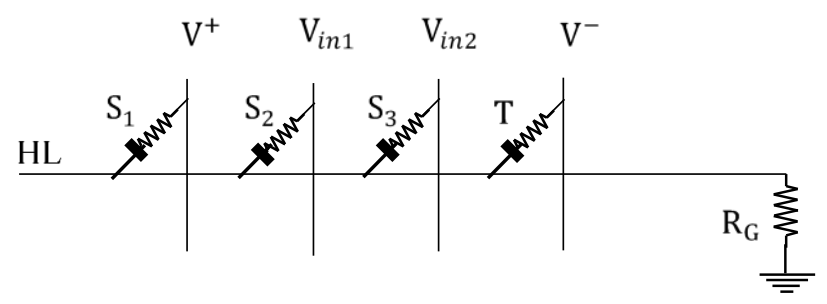

Fig. 7 Mixed-input NOR. The implementation of three-input one-output NOR gate. The resistive input is stored in $\mathrm{S}_{1}$ and the voltage inputs are applied to $S_{2}$ and $S_{3}$. The output is stored in memristor $\mathrm{T}$

both encoding schemes in a partitioned system. In each separate partition, one encoding scheme is utilized and partitions with different schemes communicate through inverters.

\subsection{Mixed-input logic gates in a crosspoint array}

Inputs on standard volistor gates are voltages; however as shown in Fig. 4e, implementing gates with mixed-inputs is possible using a hybrid of stateful and volistor logic where some inputs are represented by resistances and some by voltages. Fig. 7 depicts a three-input NOR gate implemented in a $1 \times 4$ crosspoint array where $\left(s, \operatorname{vin}_{1}, v i n_{2}, t\right)=(0$, $0,1,1)$. In $\left(s, \operatorname{vin}_{1}, v i n_{2}, t\right), s$ and $t$ are resistive logic values of $\mathrm{S}_{1}$ and $\mathrm{T}$, and $\operatorname{vin}_{1}$ and $\operatorname{vin}_{2}$ are logic input voltages applied to $S_{2}$ and $S_{3}$, see Fig. 7. Specifically, $s$ is the resistive logic value stored in $S_{1}$ and it is to be interpreted as logic ' 0 '. Assume s has been set in a previous operation and that all memristors with voltage inputs have already been set to $\mathrm{R}_{\mathrm{CLOSED}}$. As in stateful logic, $\mathrm{HL}$ is grounded through $\mathrm{R}_{\mathrm{G}}$ and $\mathrm{V}^{+}$is applied to $\mathrm{S}_{1}$. As in volistor logic input data are applied to memristors $S_{2}$ and $S_{3}$, and bias voltage $V^{-}$is applied to memristor T. Equation (3), discussed in Section 3.1, requires $\mathrm{V}_{\text {bias }}=\mathrm{V}^{-}$and $\mathrm{V}_{\text {in }} \geq 0 \mathrm{~V}$. With this configuration, the resistive value of logic s manifests as a voltage on $\mathrm{HL}$ and the circuit operates in the same manner as a volistor NOR. Translation between logical encoding schemes is accomplished in the same manner as described in Section 3.5. Mixed volistor AND gate is realized with one extra step; since all input resistances need to be negated, one additional pulse is required.

\section{HYBRID APPROACH TO SYNTHESIZE BOOLEAN FUNCTIONS IN CROSSBAR NETWORKS}

In theory [10-11] every single-output Boolean function of $n$ variables can be realized in a crosspoint array with $n$ source memristors and two additional working memristors. These working memristors can act as both source and target memristors. However, this realization leads to long sequences of pulses. These long sequences can be avoided by the use of crossbar arrays to realize arbitrary multi-output Boolean functions. Crosspoint arrays are the building blocks of crossbar arrays. 
In this section, we first discuss hybrid computation in a crosspoint array and then we show how to implement arbitrary Boolean functions in a network of crossbar arrays. This network uses a combination of stateful, volistor and mixedinput gates. These generic network structures can be used to implement logic functions in several forms such as SOP (Sum of Product), POS (Product of Sum) and TANT (Tree level AND NOT Network) [12]. TANT architecture requires non-inverted inputs, but we consider a generalized TANT with no restriction on input polarity. In this paper, stateful operations are realized solely with NOR and NOT. Note that the NOT gate can be obtained from INH by setting the non-inverted input to constant 1 . Also, the NOR gate can be created by cascading stateful INH gates and setting the non-inverted input of the first gate of the cascade to constant 1 , e.g. $\overline{a+b}=(1 \cdot \bar{a}) \cdot \bar{b}$.

\subsection{Hybrid computation in a crosspoint array}

The simplest structure to perform memristive logic computation is a crosspoint array. In this structure, computations can be performed by two approaches: 1) solely stateful logic [1], 2) a combination of mixed-input, stateful and volistor logic. We call the second approach the hybrid approach. The first approach is potentially slow since a long sequence of operations needs to be implemented. However, the second approach has the potential to reduce the number of required operations. We assume a SOP with $M$ single literal degenerate products and $N$ products with more than one literal, thus the SOP is the OR of $M+N$ inputs. Realization of this SOP function requires a four-step process to be computed with the second approach:

(1) CLEAR: Set all memristors to the closed state. In a $1 \times n$ array, this requires driving all vertical nanowires to $\mathrm{V}^{+}$ and the horizontal nanowire to $\mathrm{V}^{-}$; for an $n \times 1$ array, the driving voltages should be swapped. This step is realized in one pulse.

(2) AND: Sequentially perform $N$ volistor AND gates, each in one pulse. This step requires a total of $N$ pulses.

(3) NOR: Perform a NOR operation on $N+M$ arguments. This includes the $M$ single literal variables, which are supplied as voltages, and the $N$ products from the previous step, which are stored as resistances. This step requires one pulse.

(4) NOT: Negate the result of the previous step. This step requires one pulse. 


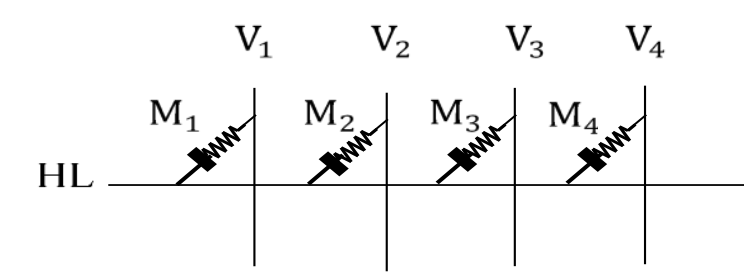

(a)

\begin{tabular}{|c|c|c|c|c|c|c|c|c|c|c|}
\hline \multirow[t]{2}{*}{ Operation } & \multirow[t]{2}{*}{ Step } & \multicolumn{5}{|c|}{$\begin{array}{l}\text { Memristors' Drivers } \\
\qquad \mathrm{V}_{i}\end{array}$} & \multicolumn{4}{|c|}{$\begin{array}{l}\text { Logic state of } \\
\text { Memristors. } \\
\mathrm{M}_{i}\end{array}$} \\
\hline & & HL & $\mathrm{V}_{1}$ & $\mathrm{~V}_{2}$ & $V_{3}$ & $\mathrm{~V}_{4}$ & $\mathrm{M}_{1}$ & $\mathrm{M}_{2}$ & $M_{3}$ & $\mathrm{M}_{4}$ \\
\hline 1 & CLEAR & $\mathrm{V}^{-}$ & $\mathrm{V}^{+}$ & $\mathrm{V}^{+}$ & $\mathrm{V}^{+}$ & $\mathrm{V}^{+}$ & 1 & 1 & 1 & 1 \\
\hline 2 & \multirow{2}{*}{ AND } & $\mathrm{HZ}$ & $\mathrm{V}_{\overline{\mathrm{a}}}$ & $\mathrm{V}_{\overline{\mathrm{b}}}$ & $\mathrm{HZ}$ & $\mathrm{V}^{-}$ & 1 & 1 & 1 & $a b$ \\
\hline 3 & & $\mathrm{HZ}$ & $\mathrm{V}_{\mathrm{a}}$ & $V_{b}$ & $\mathrm{~V}^{-}$ & $\mathrm{HZ}$ & 1 & 1 & $\bar{a} \bar{b}$ & $a b$ \\
\hline 4 & NOR & $\mathrm{R}_{\mathrm{G}}$ & $V_{c}$ & $\mathrm{~V}^{-}$ & $\mathrm{V}^{+}$ & $\mathrm{V}^{+}$ & 1 & $\bar{f}$ & $\bar{a} \bar{b}$ & $a b$ \\
\hline 5 & NOT & $\mathrm{R}_{\mathrm{G}}$ & $\mathrm{V}^{-}$ & $\mathrm{V}^{+}$ & $\mathrm{HZ}$ & $\mathrm{HZ}$ & $f$ & $\bar{f}$ & $\bar{a} \bar{b}$ & $a b$ \\
\hline
\end{tabular}

(b)

Fig. 8 Example of hybrid computation in a crosspoint array. (a) A $1 \times 4$ crosspoint array used to implement SOP function $f$. (b) The circuit configuration to implement each step. The total number of consecutive operations (pulses) to realize $f$ is 5

Example 1. The following example describes the hybrid approach in a crosspoint array for the Boolean function $f=$ $a b+\bar{a} \bar{b}+c$. This function can be realized in a $1 \times 4$ crosspoint array in five consecutive operations, one for CLEAR, two for AND, one for NOR and one for NOT, as shown in Fig. 8.

Fig. 8a shows a $1 \times 4$ crosspoint array driven by $V_{i}$ and Fig. 8 b shows the circuit configuration in each step. The first step is implemented by setting all $\mathrm{V}_{i}$ to $\mathrm{V}^{+}$and $\mathrm{HL}$ to $\mathrm{V}^{-}$. This step will set the memristors $\mathrm{M}_{i}$ closed, i.e. encoding logic ' 1 '. The second step is to compute the product $a b$. This step is implemented by setting $V_{1}$ and $V_{2}$ to $V_{\overline{\mathbf{a}}}$ and $V_{\overline{\mathrm{b}}}$ where $\mathrm{V}_{\overline{\mathrm{a}}}$ and $\mathrm{V}_{\overline{\mathrm{b}}}$ are voltage signals encoding literals $\bar{a}$ and $\bar{b}$, respectively. The result of this computation is stored in $\mathrm{M}_{4}$. Similarly, the second product, $\bar{a} \bar{b}$, is computed by setting $\mathrm{V}_{1}$ and $\mathrm{V}_{2}$ to $\mathrm{V}_{\mathrm{a}}$ and $\mathrm{V}_{\mathrm{b}}$ where $\mathrm{V}_{\mathrm{a}}$ and $\mathrm{V}_{\mathrm{b}}$ are voltage signals encoding literals $a$ and $b$, respectively. The computed result is stored in $\mathrm{M}_{3}$. The third step is realized with a mixed-input NOR gate, i.e. NOR $(a b, \bar{a} \bar{b}, c)$. Note that variable $c$ in SOP function $f$ is a voltage signal, whereas $a b$ and $\bar{a} \bar{b}$ are resistive signals. This step is implemented by connecting $\mathrm{HL}$ to the ground through $\mathrm{R}_{\mathrm{G}}$, and driving $\mathrm{V}_{1}, \mathrm{~V}_{2}, \mathrm{~V}_{3}$, and $\mathrm{V}_{4}$ to $\mathrm{V}_{\mathrm{c}}, \mathrm{V}^{-}, \mathrm{V}^{+}$, and $\mathrm{V}^{+}$, respectively. The result of the NOR gate is stored in $\mathrm{M}_{2}$. Currently, the logic value of $\mathrm{M}_{2}$ is $\bar{f}$, thus the last step is to invert this logic value to obtain $f$. This step is implemented by connecting HL to ground through $R_{G}$, and driving $V_{1}$ and $V_{2}$ to $V^{-}$and $V^{+}$, respectively. Since $M_{3}$ and $M_{4}$ do not take part in the last computation, $\mathrm{V}_{3}$ and $\mathrm{V}_{4}$ are terminated to $\mathrm{HZ}$. 
As illustrated in Example 1, every SOP can be realized in $N+3$ operations, requiring at least $N+1+M$ memristors in the crosspoint array. In Example 1, $M=1$, so we used four memristors. As a matter of proper operation, all target memristors $\mathrm{T}_{i}$ must be initially closed. Further, if any of the inputs are logic ' 1 ' then at least one of those logic ' 1 ' inputs must be driving a closed source memristor $S_{i}$. If all such memristors $S_{i}$ were open, the electrical characteristics of the memristors might prevent proper manifestation of the input voltage on the common nanowire. Therefore all memristors should be initially closed. The closed state of a memristor will only change to open when driven by $\mathrm{V}_{\text {bias }}$ and the voltage drop across the memristor is $\mathrm{V}_{\text {OPEN }}$. Therefore a target memristor $\mathrm{T}$ can be used as a source memristor,

i.e. driven by $V_{\text {in }}$ in subsequent operations with no risk of changing its state. This reuse allows for compact logic implementations. However, this approach potentially results in all source memristors being open, so any subsequent logic ' 1 ' inputs have no closed memristor to drive. Therefore the number of memristors in the array should be more than the number of products, i.e. extra memristors should be provided as dedicated targets. So, realizing volistor gates with many inputs is possible neglecting the resistance of the common nanowire. This allows implementing structures such as SOP with many products. However, in a crosspoint array, each nanowire is driven by an individual CMOS driver with control circuitry, as shown in Fig. 2. This requirement imposes a large area penalty potentially restricting the number of inputs to the gates. Extending a crosspoint array to a crossbar array overcomes this potential limitation and increases the overall memristive density.

\subsection{Hybrid computation in a crossbar array}

The crosspoint array can be scaled into a two-dimensional crossbar array of size $m \times n$. Each of the $m$ rows in the crossbar array can be thought of as a $1 \times n$ crosspoint array and each of the $n$ columns can be thought of as an $m \times 1$ crosspoint array. With this crossbar structure, in a single volistor operation a product of literals can be created and copied to an arbitrary column memristor or row memristor in the crossbar simultaneously. The multi-output capability of crosspoint arrays discussed earlier allows an arbitrary number of copy operations to be performed in any of the two dimensions of the crossbar array. Fig. 9a depicts target memristors as multi-input volistor gates whose outputs are the states of the corresponding memristors. Using the multi-output property, any combination of NOR/AND gates can be copied to any number of arbitrary locations in a single column. One operation is required for each gate type. In the worst case, the entire crossbar array can be populated with an arbitrary combination of gates in $2 n$ operations. Populating the crossbar array in this manner is the first step (after initialization) to map a Boolean function to a generic 


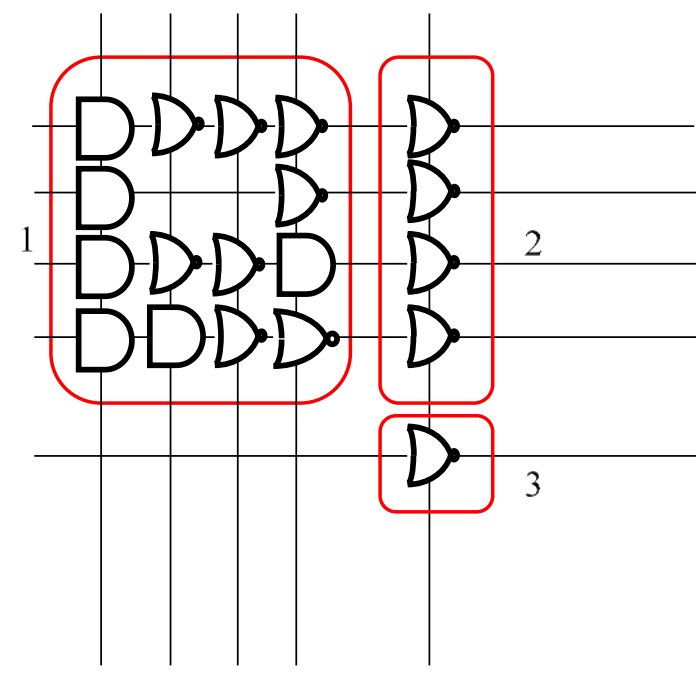

(a)

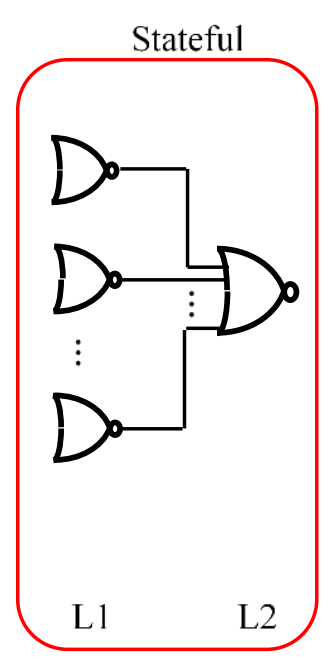

(b)

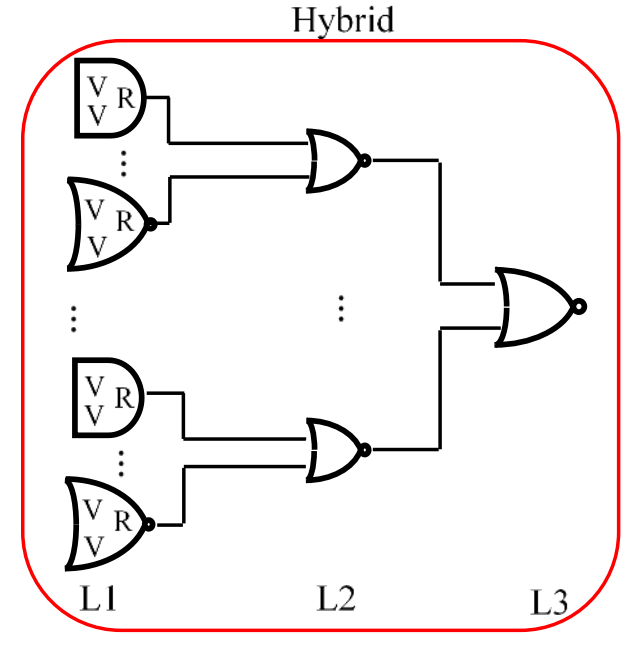

(c)

Fig. 9 The crossbar array. (a) A crossbar array divided into three blocks; the populated gates in the block labeled 1 are realized by volistors, but the blocks labeled 2 and 3 are realized with stateful NOR. (b) The symbolic two-level circuit is realized with the stateful approach. (c) The symbolic three-level circuit is realized with the hybrid approach. The first level of the circuit, L1 is realized with volistor logic, whereas the next levels L2 and L3 are realized with the stateful approach

crossbar fabric. This fabric may, in general, combine the use of volistor logic, mixed input logic and stateful logic in the same crossbar array to produce logic computations.

Example 2. As a means of discussing this hybrid approach, take for example, the implementation of function $f=$ $\overline{\overline{a \bar{b}+\bar{c} \bar{d}}+\bar{A}}=\overline{\bar{B}+\bar{A}}=A B$. This implementation requires the same four-step process described in Section 4.1 and is illustrated in Fig. 10. The entries of the symbolic matrices represent the logic values stored in each memristor of the crossbar. Voltages applied to the horizontal and vertical nanowires are indicated by the values shown to the left of and on top of the matrices. The labels on top of the arrows between matrices indicate the number of operations required to move to the next matrix. Fig. 10a shows the crossbar after initialization; the voltages to achieve this state are not shown. Fig. 10b shows the result of computing the first product with volistor AND. Fig. 10c shows the computation of the other product which is also realized with volistor AND. Fig. 10d shows stateful logic being used to compute $\bar{B}=$ $\overline{a \bar{b}+\bar{c} \bar{d}}$. In this step, $\mathrm{V}^{+}$and $\mathrm{V}^{-}$are equal to $\mathrm{V}_{\mathrm{COND}}$ and $\mathrm{V}_{\mathrm{SET}}$, and $\mathrm{R}_{\mathrm{G}}$ is the load resistor in stateful INH [2]. Fig. 10e shows the implementation of a mixed NOR gate with resistance-based signal $\bar{B}$ and voltage-based signal $\bar{A}$ producing the desired function $f$.

With the hybrid approach, the use of stateful IMP can be avoided. This simplifies the driver circuitry, removes the need for a keeper circuit [13], and simplifies crossbar initialization since all memristors are initialized as closed. The 
stateful approach also requires a step to store the inputs in the crossbar. However, since the hybrid approach uses voltages as inputs no storage step is required. The main advantage of the hybrid approach over the stateful approach is that, for the same number of operations and memristors, one additional logic level can be realized. The hybrid approach uses the efficiency of volistors to implement the first level of logic which is usually most complex. Fig. 9a depicts a crossbar array divided into three blocks. In stateful logic the memristors in block 1 store inputs; the memristors in block 2 store the first-level logic outputs, and the memristor in block 3 stores the output of the secondlevel logic. In the hybrid approach, since inputs are voltages, the memristors in block 1 store the outputs of the firstlevel logic; the memristors in block 2 store the outputs of the second-level logic, and the memristor in block 3 stores the output of the third-level logic. With the same number of operations and the same number of memristors the stateful approach produces only two-level logic (Fig. 9b) whereas the hybrid approach produces three-level logic as shown in Fig. 9c. However, since in volistor logic the inputs are voltages, there can be only one set of inputs applied to the crossbar at any given moment and therefore only one output can be computed at a time. In stateful logic, inputs are stored as resistances. Therefore each row or column can be thought of as a distinct set of inputs capable of simultaneously producing distinct outputs, with the restriction that all outputs implement the same type of stateful gate [2], e.g. all the four NOR gates as shown in block 2 of Fig. 9a. In Section 4.3, we use the hybrid approach in a crossbar network to achieve different outputs in parallel.

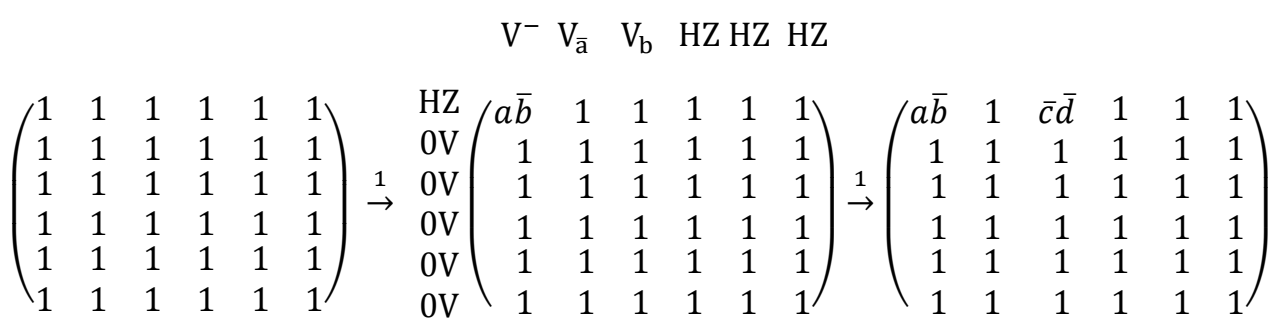

(a)

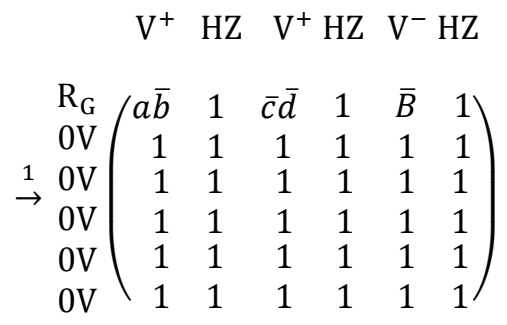

(d) (b)

(c)

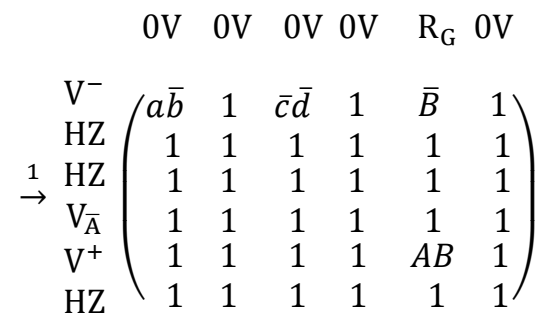

(e)

Fig. 10 The symbolic matrices illustrate the steps of logic computations based on the hybrid approach for computing $f=\overline{\bar{a} \bar{b}+\bar{c} \bar{d}+\bar{A}}$. (a) Initialization step. (b) Computing $a \bar{b}$ with a volistor AND. (c) Computing $\bar{c} \bar{d}$ with a volistor AND. (d) Computing $\overline{a \bar{b}}+\bar{c} \bar{d}$ with a stateful NOR. (e) Computing $f$ with a mixed-gate NOR 
Table 3 Different forms of logic functions and their De Morgan's equivalent

\begin{tabular}{|c|c|l|l|c|}
\hline$\#$ & Logical Form & $\begin{array}{l}\text { De Morgan's } \\
\text { Equivalent }\end{array}$ & Example & $\begin{array}{l}\text { \# of } \\
\text { Pulses }\end{array}$ \\
\hline 1 & AND-OR (SOP) & AND-NOR-NOT & Fig. 11a & 3 \\
\hline 2 & OR-AND (POS) & NOR-NOR & Fig. 11b & 2 \\
\hline 3 & $\begin{array}{c}\text { NAND-NAND- } \\
\text { NAND }\end{array}$ & AND-NOR-NOR-NOT & Fig. 11c & 4 \\
\hline 4 & $\begin{array}{c}\text { AND-EXOR } \\
\text { (ESOP) }\end{array}$ & AND-NOT-NOR-NOR & Fig. 11d & 4 \\
\hline 5 & $\begin{array}{c}\text { NAND-AND- } \\
\text { EXOR }\end{array}$ & $\begin{array}{l}\text { AND-NOR-NOT-NOR- } \\
\text { NOR }\end{array}$ & Fig. 11e & 5 \\
\hline 6 & AND-EXOR-OR & $\begin{array}{l}\text { AND-NOT-NOR-NOR- } \\
\text { NOR-NOT }\end{array}$ & Fig. 11f & 7 \\
\hline
\end{tabular}

\subsection{Hybrid computation in a crossbar network}

In a data path of a larger memristor architecture there are usually several combinational blocks as well as memories each of which is realized in one or more crossbars. A question arises as to how one can implement information transfer between these crossbars. Let us consider transfer of data from source memory to target combinational logic. The memories can store information as either voltage (e.g. CMOS memory) or as resistance. If data are stored as voltage, the first level of the target logic can be implemented with volistors. However, when data in memories are stored as resistances, the first logic level in combinational logic is realized with stateful NOR/NOT. Another problem is how to partition large combinational blocks to improve processing time by increasing parallelism. In a single crossbar array using volistor logic, identical gates with identical inputs can be produced in a single step. This is a limited form of parallelism that replaces fan-out. This limitation can be resolved using a network of many individual crossbars. Each individual crossbar can have as many copies as necessary of a single gate with identical inputs. This structure allows two or more separate crossbars to simultaneously calculate the first logic level of an arbitrary Boolean function. Additional logic levels can be achieved with stateful operations, mixed-input gates, or both. Table 3 shows different structures to realize Boolean functions that are well suited to this hybrid approach. Next, we present detailed examples for cases 2 and 5 from Table 3.

Example 3: POS implementation. The three variable EXOR expression $f=a \oplus b \oplus c$ can be implemented as the POS expression $(a+b+c)(a+\bar{b}+\bar{c})(\bar{a}+\bar{b}+c)(\bar{a}+b+\bar{c})$. The De Morgan equivalent expression is $f=$ $\overline{\overline{(a+b+c)}+\overline{(a+\bar{b}+\bar{c})}+\overline{(\bar{a}+\bar{b}+c)}+\overline{(\bar{a}+b+\bar{c})}}=\overline{w+x+y+z}$. The function is synthesized in four crossbar arrays each of which is $4 \times 4$. In this crossbar column, adjacent crossbars communicate through vertical switches capable of connecting and disconnecting the crossbars as illustrated in Fig. 12a. Fig. 12b shows the crossbar 
column with closed vertical switches between all adjacent crossbars creating a 16×4 crossbar array. These switches are not further discussed in this work. Executing $f$ is a three-step procedure.

(1) Initialize all crossbar arrays by driving all vertical nanowires with $\mathrm{V}^{+}$and all horizontal nanowires with $\mathrm{V}^{-}$.

(2) Simultaneously compute volistor NOR of each maxterm in a separate crossbar column as described in Section

3.3 3.3. In Fig. 12a, individual crossbar arrays A, B, C and D compute $w=\overline{a+b+c}, x=\overline{a+\bar{b}+\bar{c}}, y=\overline{\bar{a}+\bar{b}+c}$ and $z=\overline{\bar{a}+b+\bar{c}}$ in parallel.

(3) Connect the vertical switches between adjacent crossbars to form a $16 \times 4$ crossbar array. Perform a stateful NOR on the logic values computed in step 2 as illustrated in Fig. 12b.

Executing $f$ with a solely stateful approach would require two steps plus the overhead of setting up each input [2].

This input overhead is directly proportional to the number of inputs in the largest sum. Volistor logic has no such input

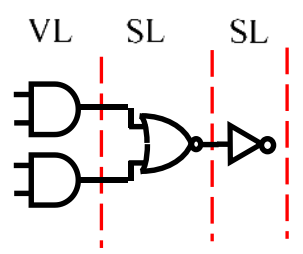

$1 \mathrm{P} \quad 1 \mathrm{P} \quad 1 \mathrm{P}$

(a)

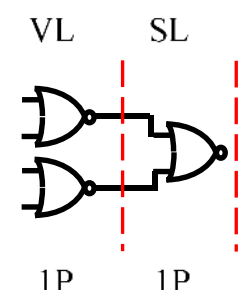

(b)

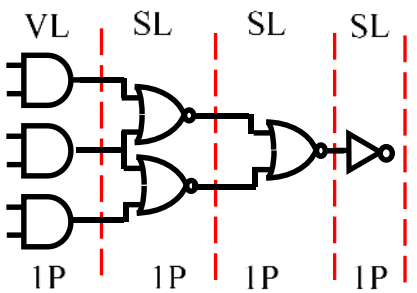

(c)

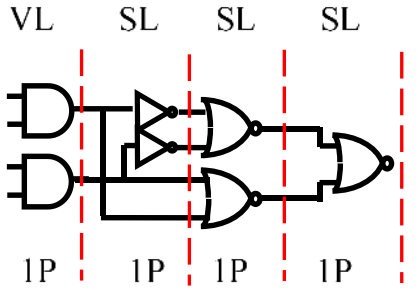

(d)

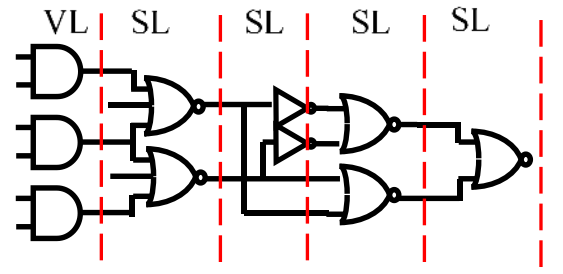

$1 \mathrm{P}$ $1 \mathrm{P}$ $1 \mathrm{P}$ IP $1 \mathrm{P}$ (e)

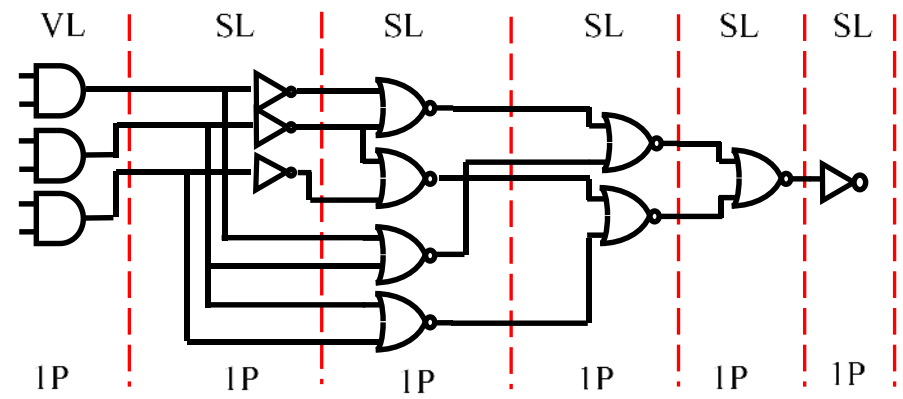

(f)

Fig.11 Implementation of different forms of Boolean functions with the hybrid approach. (a) An example of a SOP function. (b) An example of a POS function. (c) An example of a three level sum of products of sums. (d) An example of an EXOR of two products. (e) An example of a NAND-AND-EXOR logic function. (f) An example of an AND-EXOR-OR logic function. P stands for pulse (operation), e.g. 1P indicates that a related logic level requires one pulse to be implemented. VL and SL stand for volistor and stateful operations. In all circuits, only the first logic level is implemented with volistor logic 


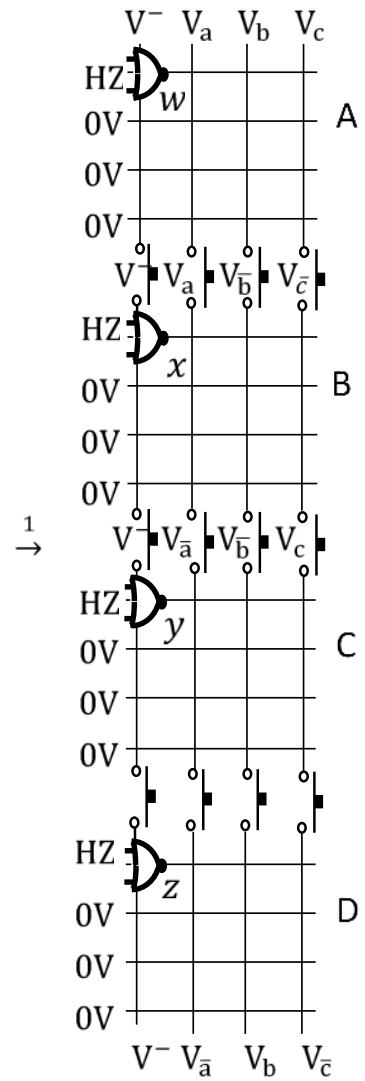

(a)

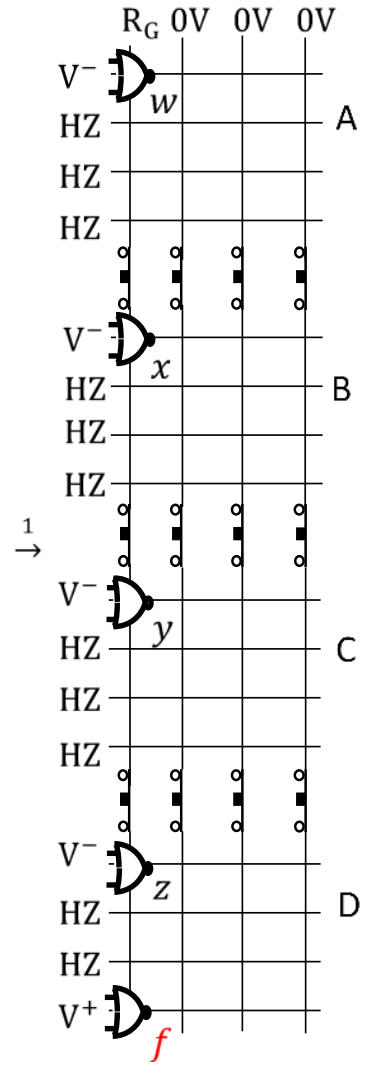

(b)

Fig. 12 Implementation of POS function $\mathrm{f}$ in a crossbar network comprised of four crossbar arrays of size $4 \times 4$. The function is realized in a two-step procedure. (a) Realization of the first logic level of POS fucntion $f$ in separate crossbars. The network configuration shows the voltage drivers applied to each nanowire. This step produces four NOR gates. (b) The switches between the $4 \times 4$ arrays are closed to create a $16 \times 4$ crossbar array. The results $w, x, y$, and $z$ of the first step are NORed to create the output of the POS function $f$

overhead; a multi-input gate and a single-input gate are both produced in the same number of steps. However, the solely stateful approach can be executed in two steps in a single crossbar whereas the hybrid approach would require more steps if performed in a single crossbar.

Example 4: NAND-AND-EXOR implementation. Consider the expression $f=e \cdot \overline{\bar{a} b} \cdot \overline{\bar{c} d} \oplus \overline{\bar{c} d} \cdot \overline{a e} \cdot b$. The De

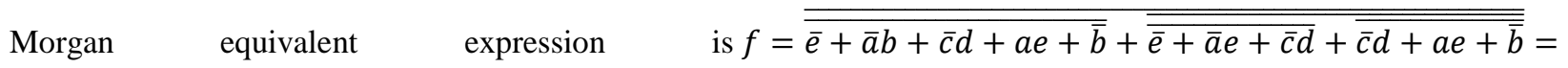

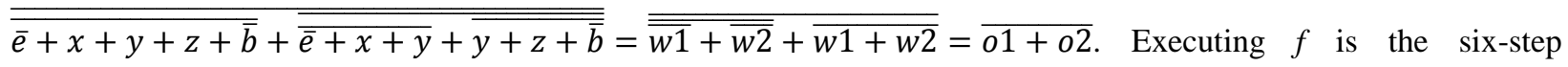
procedure depicted in Fig. 13 and described as follows. In each step, all outputs are computed simultaneously.

(1) Perform initialization as in Example 3.

(2) Compute the products $x, y$ and $z$ in parallel. Value $y$ is computed twice to enable simultaneous computation in step 3 as in Fig. 13a. This realizes the four AND gates using volistors. 
(3) Using mixed-input gates, as described in Section 3.6, compute $w 1$ and $w 2$ where $V_{\bar{e}}$ and $V_{\bar{b}}$ are input voltages and $x, y$, and $z$ are input resistances, as in Fig. 13b. This realizes the NOR logic level.

(4) Produce $\overline{w 1}$ and $\overline{w 2}$ in parallel, as in Fig. 13c. This performs two stateful NOT operations in the third logic level.

(5) Produce $o 2$ and $o 1$ in the fourth logic level, NOR, using the outputs calculated in steps 3 and 4, as in Fig. 13d.

(6) Produce $f$ from $o 2$ and $o 1$, as in Fig. 13e. This produces the fifth level of logic, NOR. Executing $f$ with a solely stateful approach would require six steps plus the input setup overhead. Note that NAND-AND-EXOR circuits are a new concept in logic synthesis. These circuits are a generalization of the PSE circuits introduced in [14] in which only one argument of the AND layer is a NAND and others are literals.

\section{COMPARISON OF POWER CONSUMPTION AND SPEED BETWEEN STATEFUL AND VOLISTOR CIRCUIT MODELS}

In this work, power measurements are made using the same model mentioned in Section 2 in the LTSpice IV simulation environment. The average power $\mathrm{P}_{\mathrm{avg}}$ consumed in a crosspoint array is computed using the equation $P_{\text {avg }}=\sum V_{R M S} \cdot I_{R M S}$ where $V_{\text {RMS }}$ and $I_{R M S}$ are the root mean squares of the voltage across and the current through each memristor. The average power consumption of each individual element of a $1 \times n$ crosspoint array where $n \leq 64$ is computed over a 10ns interval beginning with the application of the driver voltages $\mathrm{V}^{+}=0.6 \mathrm{~V}, \mathrm{~V}^{-}=-0.6 \mathrm{~V}$ and 0 Vwhich are applied for $8 \mathrm{~ns}$. Let the power consumed in a source memristor set to logic ' 1 ' be denoted $\mathrm{P}_{\mathrm{S} 1}$, the power consumed in a source memristor set to logic ' 0 ' be denoted $\mathrm{P}_{\mathrm{S} 0}$, the power consumed in a target memristor be denoted $\mathrm{P}_{\mathrm{T}}$ and the power consumed in load resistor $\mathrm{R}_{\mathrm{G}}$ be denoted $\mathrm{P}_{\mathrm{RG}}$. Let $\mathrm{P}_{\mathrm{S}}$ denote the total power consumption in all source memristors. The superscripts SL and VL are used to indicate power consumptions during stateful logic and volistor logic, respectively. For example, $\mathrm{P}_{\mathrm{S} 0}^{\mathrm{SL}}$ is the power consumed by a source memristor set to logic ' 0 ' when $\mathrm{SL}$ is performed and $\mathrm{P}_{\mathrm{S}}^{\mathrm{VL}}$ is the power consumed in all source memristors when volistor logic is performed. Table 4 describes the power consumption in each crosspoint element where $\mathrm{V}_{\mathrm{HL}}$ and $\widehat{\mathrm{V}}_{\mathrm{HL}}$ are the voltages on the horizontal nanowire during VL and SL operations, respectively. Table 4 is derived based on the average power equation $\mathrm{P}_{\mathrm{avg}}$ and Ohm's Law; the circuit elements considered in power computation are the resistance values of the memristors, either $\mathrm{R}_{\text {CLOSED }}$ or $\mathrm{R}_{\mathrm{OPEN}}$, load resistance $\mathrm{R}_{\mathrm{G}}$, and the driver voltages. The power required to connect the nanowires to $\mathrm{V}^{+}$, $\mathrm{V}^{-}, 0 \mathrm{~V}, \mathrm{R}_{\mathrm{G}}$ and $\mathrm{HZ}$ is the same in both $\mathrm{VL}$ and $\mathrm{SL}$ operations and is not considered in this work. The resistance of the horizontal nanowire $\mathrm{HL}$ is negligible when compared with load resistance $\mathrm{R}_{\mathrm{G}}$. Jo et al. [15] reported that the resistive 


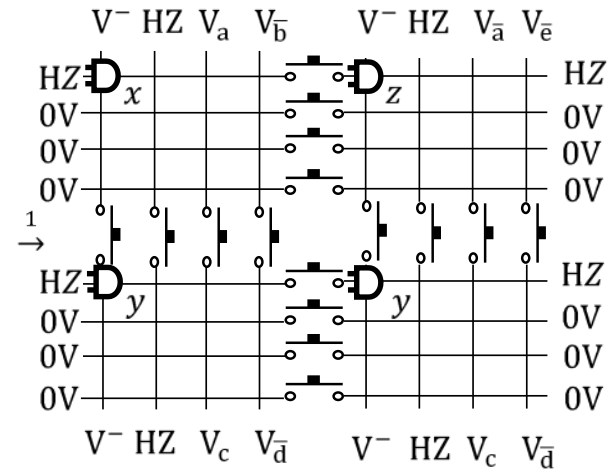

(a)

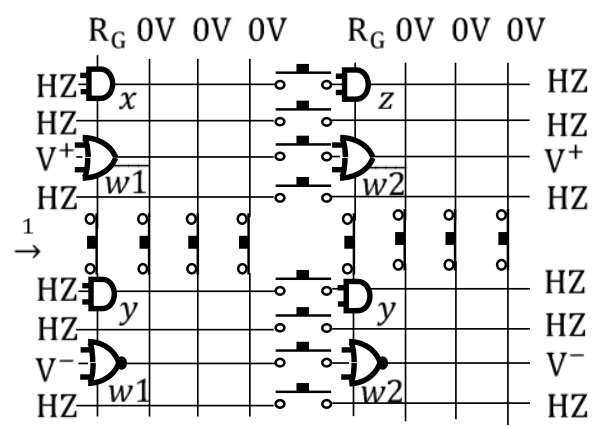

(c)

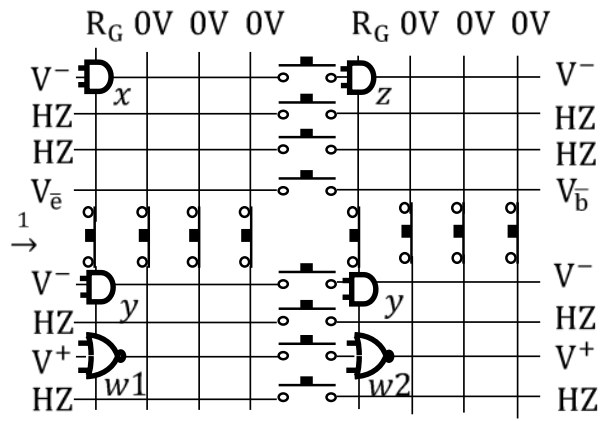

(b)

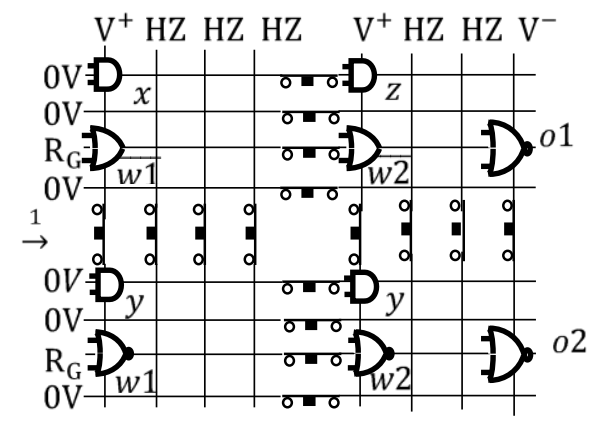

(d)

$0 \mathrm{~V} 0 \mathrm{~V} \mathrm{OV} \mathrm{R}_{\mathrm{G}}$

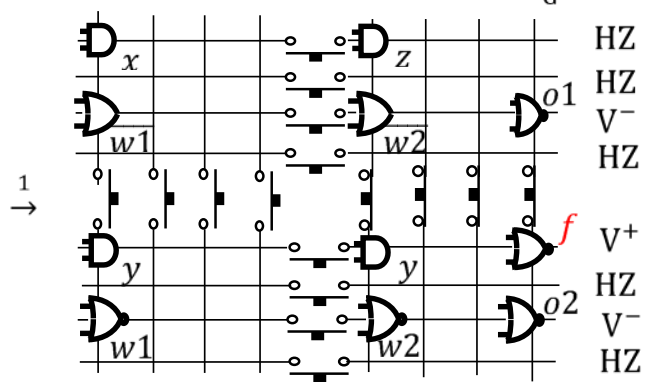

(e)

Fig. 13 Realization of the De Morgan equivalent for the NAND-AND-EXOR expression $f$. The crossbar network is comprised of four crossbar arrays each of size $4 \times 4$. The function is realized in a six-step procedure. (a) The first logic level of $f$ is realized with volistors, (b) the second logic level of $f$ is realized with mixed-input gates, and (d)-(e) the rest of the logic levels are realized with stateful logic. Crossbar drivers are indicated by $0 \mathrm{~V}, \mathrm{~V}^{+}, \mathrm{V}^{-}, \mathrm{R}_{\mathrm{G}}$, and $\mathrm{HZ}$. In each step, the performed operation is depicted by logic gates and the results of the operations are shown as outputs of the gates

value of a relatively large width nanowire of diameter $120 \mathrm{~nm}$ used in a $1 \mathrm{~Kb}$ crossbar array is at most $30 \mathrm{~K} \Omega$ when implemented with relatively high resistance p-doped $\mathrm{Si}$. This upper bound is much smaller than $\mathrm{R}_{\mathrm{G}}=15 \mathrm{M} \Omega$ chosen in this work. Recall, $\mathrm{R}_{\mathrm{G}}=\sqrt{\mathrm{R}_{\mathrm{OPEN}} \times \mathrm{R}_{\mathrm{CLOSED}}}$ and $\frac{\mathrm{R}_{\mathrm{OPEN}}}{\mathrm{R}_{\mathrm{CLOSED}}}=10^{3}$ as mentioned in Section 2 and Section 3. The power consumed by volistor logic in a crosspoint array is entirely due to the leakage through the reverse biased rectifying memristors as there is no direct path to ground. However, when volistor logic is performed in a crossbar array there is 
Table 4 Switching power consumption in each crosspoint array element

\begin{tabular}{|c|c|c|c|c|}
\hline $\begin{array}{c}\text { Logical } \\
\text { Op. }\end{array}$ & $\mathrm{P}_{\mathrm{S} 0}$ & $\mathrm{P}_{\mathrm{S} 1}$ & $\mathrm{P}_{\mathrm{T}}$ & $\mathrm{P}_{\mathrm{RG}}$ \\
\hline $\mathrm{VL}$ & $\frac{\left(\mathrm{V}_{\mathrm{HL}}\right)^{2}}{\mathrm{R}_{\mathrm{OPEN}}}$ & $\frac{\left(\mathrm{V}^{+}-\mathrm{V}_{\mathrm{HL}}\right)^{2}}{\mathrm{R}_{\mathrm{OPEN}} \times 10^{-3}}$ & $\frac{\left(\mathrm{V}^{+}+\mathrm{V}_{\mathrm{HL}}\right)^{2}}{\mathrm{R}_{\mathrm{OPEN}}}$ & 0 \\
\hline $\mathrm{SL}$ & $\frac{\left(\mathrm{V}^{+}-\widehat{\mathrm{V}}_{\mathrm{HL}}\right)^{2}}{\mathrm{R}_{\mathrm{OPEN}}}$ & $\frac{\left(\mathrm{V}^{+}-\widehat{\mathrm{V}}_{\mathrm{HL}}\right)^{2}}{\mathrm{R}_{\mathrm{OPEN}} \times 10^{-3}}$ & $\frac{\left(\mathrm{V}^{+}+\widehat{\mathrm{V}}_{\mathrm{HL}}\right)^{2}}{\mathrm{R}_{\mathrm{OPEN}}}$ & $\begin{array}{c}\frac{\widehat{\mathrm{V}}_{\mathrm{HL}}{ }^{2}}{\mathrm{R}_{\mathrm{OPEN}}} \\
\times \\
10 \sqrt{10}\end{array}$ \\
\hline
\end{tabular}

Table 5 Circuit element power consumption, propagation delay and power ratio in a $1 \times 8$ cross point array

\begin{tabular}{|c|c|c|c|c|c|c|c|c|}
\hline$\left(S_{1}, S_{0}, T\right)$ & Op & $\begin{array}{c}\mathrm{P}_{\mathrm{S} 1} \\
(\mathrm{nW})\end{array}$ & $\begin{array}{c}\mathrm{P}_{\mathrm{S} 0} \\
(\mathrm{nW})\end{array}$ & $\begin{array}{c}\mathrm{P}_{\mathrm{T}} \\
(\mathrm{nW})\end{array}$ & $\begin{array}{c}\mathrm{P}_{\mathrm{RG}} \\
(\mathrm{nW})\end{array}$ & $\begin{array}{c}\mathrm{t}_{\mathrm{d}} \\
(\mathrm{ns})\end{array}$ & $\begin{array}{c}\mathrm{P}_{\text {avg }} \\
(\mathrm{nW})\end{array}$ & $\frac{\mathrm{P}_{\mathrm{SL}}}{\mathrm{P}_{\mathrm{VL}}}$ \\
\hline \multirow[t]{2}{*}{$(2,2,1)$} & VL & $2.224 \mathrm{e}-3$ & 0.555 & 2.226 & 0 & 4.044 & 3.340 & \multirow[t]{2}{*}{6.13} \\
\hline & SL & 0.168 & $0.168 \mathrm{e}-3$ & 2.192 & 17.946 & 4.239 & 20.474 & \\
\hline \multirow[t]{2}{*}{$(2,3,1)$} & $\mathrm{VL}$ & $3.471 \mathrm{e}-3$ & 0.555 & 2.225 & 0 & 4.044 & 3.897 & \multirow[t]{2}{*}{5.254} \\
\hline & SL & 0.168 & $0.168 \mathrm{e}-3$ & 2.192 & 17.947 & 4.239 & 20.476 & \\
\hline \multirow[t]{2}{*}{$(2,4,1)$} & VL & $4.993 \mathrm{e}-3$ & 0.554 & 2.224 & 0 & 4.054 & 4.450 & \multirow[t]{2}{*}{4.601} \\
\hline & SL & 0.168 & $0.168 \mathrm{e}-3$ & 2.192 & 17.947 & 4.239 & 20.476 & \\
\hline \multirow[t]{2}{*}{$(2,4,2)$} & VL & $8.868 \mathrm{e}-3$ & 0.553 & 2.221 & 0 & 4.064 & 6.672 & \multirow[t]{2}{*}{3.397} \\
\hline & SL & 0.187 & $0.187 \mathrm{e}-3$ & 2.190 & 17.911 & 4.227 & 22.666 & \\
\hline \multirow[t]{2}{*}{$(2,3,3)$} & VL & $11.22 \mathrm{e}-3$ & 0.553 & 2.220 & 0 & 4.073 & 8.341 & \multirow[t]{2}{*}{2.955} \\
\hline & SL & 0.207 & $0.207 \mathrm{e}-3$ & 2.187 & 17.876 & 4.288 & 24.645 & \\
\hline \multirow[t]{2}{*}{$(2,2,4)$} & VL & 0.014 & 0.552 & 2.219 & 0 & 4.083 & 10.008 & \multirow[t]{2}{*}{2.701} \\
\hline & SL & 0.229 & $0.229 \mathrm{e}-3$ & 2.185 & 17.838 & 4.288 & 27.036 & \\
\hline
\end{tabular}

an additional power consumption in memristors not taking part in the operations. This is true for stateful logic as well.

Since the power consumption in both cases is the same and we are only interested in comparing SL and VL, this additional power is not discussed. In this section, the power consumption in a crosspoint array for $S_{1}>0$ and $S_{1}=0$ is analyzed separately. For ease of reference, let $S_{1}$ and $S_{0}$ denote the numbers of source memristors set to vin $=1$ and $\operatorname{vin}=0$, respectively; $T$ denotes the number of target memristors; $\mathrm{P}_{\mathrm{SL}}$ and $\mathrm{P}_{\mathrm{VL}}$ denote the total power consumption in memristors and in load resistor $\mathrm{R}_{\mathrm{G}}$ during SL and VL operations, respectively; and $\mathrm{t}_{\mathrm{d}}$ denotes the switching delaythe time required to completely switch from the close state of a target memristor $\mathrm{T}$ to the open state.

\subsection{Analysis of power consumption and propagation delays in a $1 \times 8$ crosspoint array for $S_{1}>0$}

Table 5 compares the switching delay, $\mathrm{t}_{\mathrm{d}}$, and the overall power consumption in memristors and in load resistor $\mathrm{R}_{\mathrm{G}}$ during SL and VL operations for various compositions of $S_{1}, S_{0}$ and $T, \frac{\mathrm{P}_{\mathrm{SL}}}{\mathrm{P}_{\mathrm{VL}}}$. From the simulation results shown in Table 5, the following is observed. 
1) In $\mathrm{SL}$ and $\mathrm{VL}, \mathrm{P}_{\mathrm{T}}$ is approximately $2 \mathrm{nW}$, i.e. the changes to the number and the high/low composition of input values slightly affect the power consumed by any individual target memristor T. This is expected given the characteristics of the rectifying memristors and considering that $\mathrm{V}_{\mathrm{HL}} \approx \mathrm{V}_{\text {in }}$.

2) The major contributor to the power consumption in $S L$ is $R_{G}-$ it consumes approximately 8 times more power than each target memristor. In VL power consumption, $\mathrm{P}_{\mathrm{RG}}=0$.

3) The minor contributors to the power consumption in $\mathrm{SL}$ is $\mathrm{S}_{0} \times \mathrm{P}_{\mathrm{S} 0}^{\mathrm{SL}}$.

4) The major contributors to the power consumption in VL are determined based on $S_{0}$ and $T$.

5) For any combination of $S_{1}, S_{0}$, and $T, \frac{\mathrm{P}_{\mathrm{SL}}}{\mathrm{P}_{\mathrm{VL}}}>2$. The lower bound of $\frac{\mathrm{P}_{\mathrm{SL}}}{\mathrm{P}_{\mathrm{VL}}}$ obtained for the composition of $\left(S_{1}, S_{0}\right.$ $, T)=(1,0,7)$ is 2.129. 6) For any combination of $S_{1}, S_{0}$, and $T, \mathrm{t}_{\mathrm{d}}$ is always lower for VL.

As a summary, we observed that in a $1 \times 8$ crosspoint array, VL circuit is faster than SL and consumes at most half the power of its SL technology equivalent.

In a $1 \times 8$ crosspoint array, for any composition of $S_{1}, S_{0}$, and $T$, where $S_{1}>0$, the power ratio can be approximated based on the following power properties, Property 1- Property 4.

Property 1. When $S_{1}>0$, the power consumption in a target memristor during VL operation can be approximated as in (5).

$\mathrm{P}_{\mathrm{T}}^{\mathrm{VL}} \approx 4 \times \mathrm{P}_{\mathrm{S} 0}^{\mathrm{VL}}$

Proof: The power consumption in a target memristor during VL operation is $\mathrm{P}_{\mathrm{T}}^{\mathrm{VL}}=\frac{\left(\mathrm{V}_{\mathrm{HL}}-\mathrm{V}^{-}\right)^{2}}{\mathrm{R}_{\mathrm{OPEN}}}=\frac{\left(\mathrm{V}_{\mathrm{HL}}+\mathrm{V}^{+}\right)^{2}}{\mathrm{R}_{\mathrm{OPEN}}}$ and the power consumption in a source memristor set to logic ' 0 ' during VL operation is $\mathrm{P}_{\mathrm{S} 0}^{\mathrm{VL}}=\frac{\mathrm{V}_{\mathrm{HL}}{ }^{2}}{\mathrm{R}_{\mathrm{OPEN}}}$. So, $\frac{\mathrm{P}_{\mathrm{T}}^{\mathrm{VL}}}{\mathrm{P}_{\mathrm{S} 0}^{\mathrm{VL}}}=\frac{\left(\mathrm{V}_{\mathrm{HL}}+\mathrm{V}^{+}\right)^{2}}{\mathrm{~V}_{\mathrm{HL}}{ }^{2}}$. Let $\mathrm{V}^{+}=\mathrm{V}_{\mathrm{HL}}+\varepsilon$. As a result, $\frac{\mathrm{P}_{\mathrm{T}}^{\mathrm{VL}}}{\mathrm{P}_{\mathrm{S} 0}}=\left[\frac{2 \mathrm{~V}_{\mathrm{HL}}+\varepsilon}{\mathrm{V}_{\mathrm{HL}}}\right]^{2}=\left[2+\frac{\varepsilon}{\mathrm{V}_{\mathrm{HL}}}\right]^{2}$. The upper and lower bounds of $\frac{\mathrm{P}_{\mathrm{T}}^{\mathrm{VL}}}{\mathrm{P}_{\mathrm{S} 0}}$, obtained for the upper and lower bounds of $\frac{\varepsilon}{\mathrm{V}_{\mathrm{HL}}}$, are calculated for $\left(S_{1}, S_{0}, T\right)=(1,1,6)$ and $\left(S_{1}, S_{0}, T\right)=(6,1,1)$, respectively. These compositions were simulated using LTSPICE obtaining $4.002 \leq \frac{\mathrm{PT}_{\mathrm{T}}^{\mathrm{VL}}}{\mathrm{P}_{\mathrm{S} 0}^{\mathrm{VL}}} \leq 4.052$. Therefore, for any combination of $S_{1}$, $S_{0}$, and $T$ where $S_{1}>0$, we can assume $\mathrm{P}_{\mathrm{T}}^{\mathrm{VL}} \approx 4 \times \mathrm{P}_{\mathrm{S} 0}^{\mathrm{VL}}$.

Property 2. When $S_{1}>0$, the power consumption in the load resistor can be approximated as in (6).

$\mathrm{P}_{\mathrm{RG}} \approx 8 \times \mathrm{P}_{\mathrm{T}}^{\mathrm{SL}}$ 
Proof: The power consumption in the load resistor is $P_{\mathrm{RG}}=\frac{\widehat{\mathrm{V}}_{\mathrm{HL}}{ }^{2}}{\mathrm{R}_{\mathrm{G}}}$ and the power consumption in a target memristor during SL operation is $\mathrm{P}_{\mathrm{T}}^{\mathrm{SL}}=\frac{\left(\widehat{\mathrm{V}}_{\mathrm{HL}}-\mathrm{V}^{-}\right)^{2}}{\mathrm{R}_{\mathrm{OPEN}}}=\frac{\left(\widehat{\mathrm{V}}_{\mathrm{HL}}+\mathrm{V}^{+}\right)^{2}}{\mathrm{R}_{\mathrm{OPEN}}}$. So, $\frac{\mathrm{P}_{\mathrm{RG}}}{\mathrm{P}_{\mathrm{T}}^{\mathrm{SL}}}=\frac{\widehat{\mathrm{V}}_{\mathrm{HL}}{ }^{2}}{\mathrm{R}_{\mathrm{G}}} \times \frac{\mathrm{R}_{\mathrm{OPEN}}}{\left.\widehat{(}_{\mathrm{HL}}+\mathrm{V}^{+}\right)^{2}}=\frac{\mathrm{R}_{\mathrm{OPEN}}}{\mathrm{R}_{\mathrm{G}}} \times \frac{1}{\left[\frac{\widehat{V}_{\mathrm{HL}}+\mathrm{V}^{+}}{\widehat{V}_{\mathrm{HL}}}\right]^{2}}$. Let $\mathrm{V}^{+}=$ $\widehat{\mathrm{V}}_{\mathrm{HL}}+\hat{\varepsilon}$. As a result, $\frac{\mathrm{P}_{\mathrm{RG}}}{\mathrm{P}_{\mathrm{T}}^{\mathrm{SL}}}=\frac{\mathrm{R}_{\mathrm{OPEN}}}{\mathrm{R}_{\mathrm{G}}} \times \frac{1}{\left[\frac{2 \widehat{\mathrm{V}}_{\mathrm{HL}}+\hat{\varepsilon}}{\widehat{\mathrm{V}}_{\mathrm{HL}}}\right]^{2}}=\frac{\mathrm{R}_{\mathrm{OPEN}}}{\mathrm{R}_{\mathrm{G}}} \times \frac{1}{\left[2+\frac{\hat{\varepsilon}}{\hat{\mathrm{V}}_{\mathrm{HL}}}\right]^{2}}$. Computing the upper bound of $\frac{\mathrm{P}_{\mathrm{RG}}}{\mathrm{P}_{\mathrm{T}}^{\mathrm{SL}}}$ requires computing the lower bound of voltage ratio $\frac{\hat{\varepsilon}}{\widehat{\mathrm{V}}_{\mathrm{HL}}}$. The upper and lower bounds of $\frac{\mathrm{P}_{\mathrm{RG}}}{\mathrm{P}_{\mathrm{T}}^{\mathrm{SL}}}$ or the lower and upper bounds of $\frac{\widehat{\varepsilon}}{\widehat{V}_{\mathrm{HL}}}$ are calculated for the compositions of $\left(S_{1}, S_{0}, T\right)=(7,0,1)$ and $\left(S_{1}, S_{0}, T\right)=(1,0,7)$, respectively. These compositions were simulated using LTSPICE obtaining $7.542 \leq \frac{\mathrm{P}_{\mathrm{RG}}}{\mathrm{P}_{\mathrm{T}}^{\mathrm{SL}}} \leq 7.866$. Therefore for any combination of $S_{1}$, $S_{0}$, and $T$ where $S_{1}>0$, we can assume $\mathrm{P}_{\mathrm{RG}} \approx 8 \times \mathrm{P}_{\mathrm{T}}^{\mathrm{SL}}$.

Since $\mathrm{P}_{\mathrm{T}}^{\mathrm{VL}}$ and $\mathrm{P}_{\mathrm{T}}^{\mathrm{SL}}$, for any combination of $S_{1}, S_{0}$, and $T$ where $S_{1}>0$, are approximately $2 \mathrm{nW}$, they can be replaced with $\mathrm{P}_{\mathrm{T}}$ in (5) and (6), i.e. $\mathrm{P}_{\mathrm{T}} \approx \mathrm{P}_{\mathrm{T}}^{\mathrm{VL}} \approx \mathrm{P}_{\mathrm{T}}^{S \mathrm{~L}}$.

Property 3. When $S_{1}>0$, the power consumption in source memristors during SL operation is considerably smaller than the overall power consumption in target memristors and load resistor $\mathrm{R}_{\mathrm{G}}$ as in (7).

$\frac{\mathrm{P}_{\mathrm{S}}^{\mathrm{SL}}}{\mathrm{P}_{\mathrm{SL}}-\mathrm{P}_{\mathrm{S}}^{\mathrm{SL}}} \ll 1$

Proof: The simulation results show that the power consumption in source memristors during SL operation $\mathrm{P}_{\mathrm{S}}^{\mathrm{SL}}$ is negligible when compared to the overall power consumption in target memristors and load resistor $\mathrm{R}_{\mathrm{G}}$. The power consumption in source memristors set to logic ' 1 ' is $10^{3}$ times larger than the power consumption in source memristors set to logic ' 0 ' during SL operation, $\frac{\mathrm{P}_{\mathrm{S} 1}^{\mathrm{SL}}}{\mathrm{P}_{\mathrm{S} 0}^{\mathrm{SL}}}=10^{3}$, which can be shown as follows: $\frac{\mathrm{P}_{\mathrm{S}}^{\mathrm{SL}}}{\mathrm{P}_{\mathrm{S} 0}^{\mathrm{SL}}}=\frac{\left(\mathrm{V}^{+}-\widehat{\mathrm{V}}_{\mathrm{HL}}\right)^{2}}{\mathrm{R}_{\mathrm{CLOSED}}} \times \frac{\mathrm{R}_{\mathrm{OPEN}}}{\left(\mathrm{V}^{+}-\widehat{\mathrm{V}}_{\mathrm{HL}}\right)^{2}}=10^{3}$.

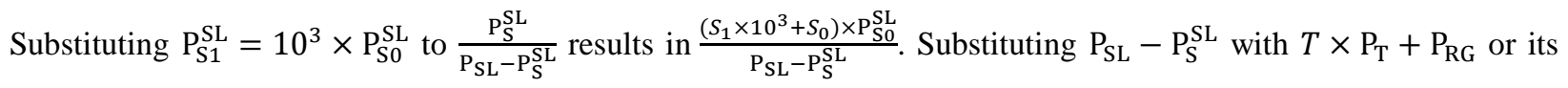
equivalent $(T+8) \times \mathrm{P}_{\mathrm{T}}$ in $\frac{\left(S_{1} \times 10^{3}+S_{0}\right) \times \mathrm{P}_{\mathrm{S} 0}^{\mathrm{SL}}}{\mathrm{P}_{\mathrm{SL}}-\mathrm{P}_{\mathrm{S}}^{\mathrm{SL}}}$ results in $\frac{\left(S_{1} \times 10^{3}+S_{0}\right)}{(T+8) \times \frac{\mathrm{P}_{\mathrm{T}}}{\mathrm{P}_{\mathrm{S} 0}^{\mathrm{SL}}}}$. It is required to compute the lower bound of $\frac{\mathrm{P}_{\mathrm{T}}^{\mathrm{SL}}}{\mathrm{P}_{\mathrm{S} 0}^{\mathrm{SL}}}$ to verify $\frac{\left(S_{1} \times 10^{3}+S_{0}\right)}{(T+8) \times \frac{\mathrm{P}_{\mathrm{T}}}{\mathrm{P}_{\mathrm{S} 0}^{\mathrm{SL}}}} \ll 1$. This power ratio $\frac{\mathrm{P}_{\mathrm{T}}}{\mathrm{P}_{\mathrm{S} 0}^{\mathrm{SL}}}$ is equal to $\frac{\left(\widehat{\mathrm{V}}_{\mathrm{HL}}-\mathrm{V}^{-}\right)^{2}}{\mathrm{R}_{\mathrm{OPEN}}} \times \frac{\mathrm{R}_{\mathrm{OPEN}}}{\left(\mathrm{V}^{+}-\widehat{\mathrm{V}}_{\mathrm{HL}}\right)^{2}}$ or $\frac{\mathrm{P}_{\mathrm{T}}}{\mathrm{P}_{\mathrm{S} 0}^{\mathrm{SL}}}=\left(\frac{2 \widehat{\mathrm{V}}_{\mathrm{HL}}}{\widehat{\varepsilon}}+1\right)^{2}$ with the substitution of $\mathrm{V}^{+}=\widehat{\mathrm{V}}_{\mathrm{HL}}+\widehat{\varepsilon}$. The lower bound of $\frac{\mathrm{P}_{\mathrm{T}}}{\mathrm{P}_{\mathrm{SO}}^{\mathrm{SL}}}$, obtained for the lower bound of $\frac{\widehat{\mathrm{V}}_{\mathrm{HL}}}{\widehat{\varepsilon}}$, is calculated for $\left(S_{1}, S_{0}\right.$, 
$T)=(1,1,6)$. This composition results in $\frac{\widehat{\mathrm{V}}_{\mathrm{HL}}}{\widehat{\varepsilon}}=21.949$ and therefore $\frac{\left(S_{1} \times 10^{3}+S_{0}\right)}{(T+8) \times \frac{P_{T}}{P_{S 0}^{S L}}}=0.036$ which is considerably smaller than 1 and thus proves (7). The immediate consequence of (7) for any combination of $S_{1}, S_{0}$, and $T$ where $S_{1}>0$ is $\mathrm{P}_{\mathrm{SL}}-\mathrm{P}_{\mathrm{S}}^{\mathrm{SL}} \approx \mathrm{P}_{\mathrm{SL}}$ used in deriving (9).

Property 4. The power consumption in source memristors driven by logic ' 1 ' during VL operation is considerably smaller than the overall power consumption in source memristors driven by logic ' 0 ' and target memristors as in (8). $\frac{S_{1} \times \mathrm{P}_{\mathrm{SL}}^{\mathrm{VL}}}{\mathrm{P}_{\mathrm{VL}}-S_{1} \times \mathrm{P}_{\mathrm{S} 1}^{\mathrm{VL}}} \ll 1$

Proof: The simulation results show that the power consumption in source memristors driven by logic ' 1 ' during VL operation is negligible when compared to the overall power consumption in memristors. This results can be proved as

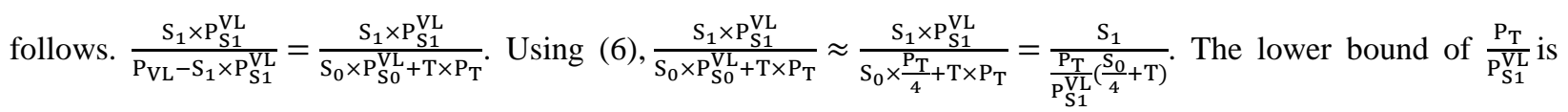
required to verify $\frac{S_{1} \times P_{S 1}^{V L}}{P_{V_{L}}-S_{1} \times P_{S 1}^{V L}} \ll 1$, and is obtained for the composition of $\left(S_{1}, S_{0}, T\right)=(1,0,7)$. Note that $\frac{\mathrm{P}_{T}}{P_{S 1}^{V L}}=$ $\frac{\left(2 \mathrm{~V}_{\mathrm{HL}}+\varepsilon\right)^{2}}{\mathrm{R}_{\mathrm{OPEN}}} \times \frac{\mathrm{R}_{\mathrm{CLOSED}}}{\left(\mathrm{V}^{+}-\mathrm{V}_{\mathrm{HL}}\right)^{2}}=10^{-3} \times\left(\frac{2 \mathrm{~V}_{\mathrm{HL}}}{\varepsilon}+1\right)^{2}$ where $\varepsilon=\mathrm{V}^{+}-\mathrm{V}_{\mathrm{HL}}$. Using LTSPICE simulator, the lower bound of $\frac{\mathrm{V}_{\mathrm{HL}}}{\varepsilon}$ for the given combination is 70.929 and consequently $\frac{\mathrm{P}_{\mathrm{T}}}{\mathrm{P}_{\mathrm{S} 1}^{\mathrm{VL}}}=20.409$. Therefore, $\frac{\mathrm{S}_{1} \times \mathrm{P}_{\mathrm{SL}}^{\mathrm{VL}}}{\mathrm{P}_{\mathrm{VL}}-\mathrm{S}_{1} \times \mathrm{P}_{\mathrm{S} 1}^{\mathrm{VL}}} \approx 0.007$ which is significantly smaller than 1 and thus proves (8). The immediate consequence of (8) for any combination of $\mathrm{S}_{1}, \mathrm{~S}_{0}$, and $\mathrm{T}$ where $\mathrm{S}_{1}>0$ is $\mathrm{P}_{\mathrm{VL}}-\mathrm{S}_{1} \times \mathrm{P}_{\mathrm{SL}}^{\mathrm{VL}} \approx \mathrm{P}_{\mathrm{VL}}$ used in deriving (9).

According to (7) and (8), $\frac{\mathrm{P}_{\mathrm{SL}}}{\mathrm{P}_{\mathrm{VL}}} \approx \frac{T \times \mathrm{P}_{\mathrm{T}}+\mathrm{P}_{\mathrm{RG}}}{S_{0} \times \mathrm{P}_{\mathrm{S} \mathrm{L}}+T \times \mathrm{P}_{\mathrm{T}}}$, and according to (5) and (6), $\frac{T \times \mathrm{P}_{\mathrm{T}}+\mathrm{P}_{\mathrm{RG}}}{S_{0} \times \mathrm{P}_{\mathrm{S} 0}^{\mathrm{VL}}+T \times \mathrm{P}_{\mathrm{T}}} \approx \frac{T \times \mathrm{P}_{\mathrm{T}}+8 \times \mathrm{P}_{\mathrm{T}}}{S_{0} \times \frac{\mathrm{P}_{\mathrm{T}}}{4}+T \times \mathrm{P}_{\mathrm{T}}}$. Therefore, for any combination of $S_{1}, S_{0}$, and $T$ where $S_{1}>0$, we can assume:

$\frac{\mathrm{P}_{\mathrm{SL}}}{\mathrm{P}_{\mathrm{VL}}} \approx \frac{T+8}{T+\frac{S_{0}}{4}}$

The lower bound of (9) is larger than 2. Simplifying the inequality $\frac{T+8}{T+\frac{S_{0}}{4}}>2$ results in $T+\frac{S_{0}}{2}<8$. In a $1 \times 8$ crosspoint array for any composition of $S_{1}, S_{0}, T$ where $S_{1}>0$, the inequality $T+\frac{S_{0}}{2}<8$ holds and so $\frac{\mathrm{P}_{\mathrm{SL}}}{\mathrm{P}_{\mathrm{VL}}}>2$.

In terms of propagation delay, volistor gates perform faster than stateful gates. The propagation delay in both SL and VL operations corresponds to the voltage drop across the target memristors or equivalently the voltage of the common nanowire during the operations. The lowest voltage drop across the target memristors is obtained for the composition of $\left(S_{1}, S_{0}, T\right)=(1,0,7)$ for which $\mathrm{t}_{\mathrm{d}}$ is $4.136 \mathrm{~ns}$ and $4.477 \mathrm{~ns}$ for VL and SL, respectively. The highest 
voltage drop across the target memristors is obtained for the composition of $\left(S_{1}, S_{0}, T\right)=(7,0,1)$ for which $\mathrm{t}_{\mathrm{d}}$ is 4.030ns and 4.089ns for VL and SL, respectively. Note that the increase of $S_{1}$ reduces the propagation delay in both SL and VL operations.

\subsection{Analysis of power consumption in a $1 \times n$ crosspoint array for $S_{1}=0$}

In a $1 \times 8$ crosspoint array, for $S_{1}=0$ and any combination of $S_{0}$ and $T$, our simulation results show that $\frac{\mathrm{P}_{\mathrm{SL}}}{\mathrm{P}_{\mathrm{VL}}}>1$.

Table 6 compares the power consumption in memristors and load resistor $\mathrm{R}_{\mathrm{G}}$ for $S_{1}=0$ and various compositions of $S_{0}$ and $T$ during VL and SL operations. In all compositions, $T$ is kept constant at 1 and $S_{0}$ is varied between 1 and 7. Table 6 shows that the increase of $S_{0}$ has almost no effect on $P_{V L}$, i.e. for all given compositions the power consumption in memristors during VL operation is almost equal to the power consumption in only target memristors.

Property 5. When $S_{1}=0$, the power consumption in source memristors driven by logic ' 0 ' during VL operation is considerably smaller than the overall power consumption in target memristors as in (10).

$\frac{S_{0} \times \mathrm{P}_{\mathrm{S} 0}^{\mathrm{VL}}}{T \times \mathrm{P}_{\mathrm{T}}^{\mathrm{VL}}} \ll 1$

Proof: Our simulation results show that the overall power consumption in memristors during VL operation is almost equal to the power consumption in only target memristors when $S_{1}=0$. The power ratio $\frac{S_{0} \times \mathrm{P}_{\mathrm{S} 0}^{\mathrm{VL}}}{T \times \mathrm{P}_{\mathrm{T}}^{\mathrm{VL}}}$ is equal to $\frac{S_{0}}{T} \times$ $\frac{\mathrm{V}_{\mathrm{HL}}{ }^{2}}{\mathrm{R}_{\mathrm{CLOSED}}} \times \frac{\mathrm{R}_{\mathrm{OPEN}}}{\left(\mathrm{V}_{\mathrm{HL}}+\mathrm{V}^{+}\right)^{2}}=10^{3}\left(\frac{S_{0}}{T}\right) \times \frac{1}{\left(1+\frac{\mathrm{V}^{+}}{\mathrm{V}_{\mathrm{HL}}}\right)^{2}}$. The upper bound of $\frac{\mathrm{P}_{\mathrm{SO}}^{\mathrm{VL}}}{\mathrm{P}_{\mathrm{T}}}$ is calculated for the composition of $\left(S_{0}, T\right)=$ $(7,1)$, i.e. this composition maximizes $\mathrm{V}_{\mathrm{HL}}$. For this composition, $\mathrm{V}_{\mathrm{HL}}=-75.417 \mu \mathrm{V}$ and thus $\frac{\mathrm{P}_{\mathrm{S}}^{\mathrm{VL}}}{\mathrm{P}_{\mathrm{T}}^{\mathrm{VL}}}=1.106 \times 10^{-4}$ which is considerably smaller than 1 and proves (10). Note that for a larger crosspoint array equation (10) is also valid, e.g. in a $1 \times 64$ crosspoint array, the upper bound of $\frac{\mathrm{P}_{\mathrm{S} 0}^{\mathrm{VL}}}{\mathrm{P}_{\mathrm{T}}^{\mathrm{VL}}}$ is calculated for $\left(S_{0}, T\right)=(63,1)$. For this composition, $\mathrm{V}_{\mathrm{HL}}=-$ $8.3807 \mu \mathrm{V}, \mathrm{P}_{\mathrm{VL}}=0.558 \mathrm{nW}$, and consequently $\frac{\mathrm{P}_{\mathrm{S}}^{\mathrm{VL}}}{\mathrm{P}_{\mathrm{T}}^{\mathrm{VL}}}=1.229 \times 10^{-5} \gg 1$. The immediate consequence of (10) for any combination of $\mathrm{S}_{0}$ and $\mathrm{T}$ is $\mathrm{P}_{\mathrm{VL}} \approx \mathrm{P}_{\mathrm{VL}}-\mathrm{S}_{0} \times \mathrm{P}_{\mathrm{S} 0}^{\mathrm{VL}}$ used in computing $\frac{\mathrm{P}_{\mathrm{SL}}}{\mathrm{P}_{\mathrm{VL}}}$. Note that for $S_{1}=0, \mathrm{P}_{\mathrm{T}}^{\mathrm{SL}} \neq \mathrm{P}_{\mathrm{T}}^{\mathrm{VL}}$ and the increase of $S_{0}$ increases the $\frac{\mathrm{P}_{\mathrm{SL}}}{\mathrm{P}_{\mathrm{VL}}}$ ratio as shown in Table 6.

Table 7 compares power ratio $\frac{\mathrm{P}_{\mathrm{SL}}}{\mathrm{P}_{\mathrm{VL}}}$ for various compositions of $S_{0}$ and $T$. In a $1 \times n$ crosspoint array, for $S_{1}=0$, the power consumption in all memristors and load resistor $\mathrm{R}_{\mathrm{G}}$ during $\mathrm{SL}$ operation for $\left(S_{0}, T\right)=(n-k, k)$ and $\left(S_{0}, T\right)=$ 
$(k, n-k)$ is the same, where $1 \leq k<n$. In other words, if the voltage on the common nanowire of the crosspoint array for $\left(S_{0}, T\right)=(n-k, k)$ is $\widehat{\mathrm{V}}_{\mathrm{HL}}$, this voltage for $\left(S_{0}, T\right)=(k, n-k)$ becomes $-\widehat{\mathrm{V}}_{\mathrm{HL}}$. For example, for $\left(S_{0}, T\right)=$ $(2,6), \widehat{\mathrm{V}}_{\mathrm{HL}}=-51.096 \mathrm{mV}$, but for $\left(S_{0}, T\right)=(6,2), \widehat{\mathrm{V}}_{\mathrm{HL}}=51.096 \mathrm{mV}$. For both compositions, $\mathrm{P}_{\mathrm{SL}}=4.246 \mathrm{nW}$, as shown in Table 7. This circuit characteristic causes $\frac{\mathrm{P}_{\mathrm{SL}}}{\mathrm{P}_{\mathrm{VL}}}$ to grow as $S_{0}$ approaches $n$. As $S_{0}$ grows, the voltage drop across the target memristors during SL operation is larger than the voltage drop across the target memristors during VL operation. Since all target memristors show the same resistance when reverse biased, $\frac{\mathrm{P}_{\mathrm{SL}}}{\mathrm{P}_{\mathrm{VL}}}>1$. Recall that in SL operation all source memristors are connected to $\mathrm{V}^{+}$, whereas in $\mathrm{VL}$ operation, under the assumption of $S_{1}=0$, all source memristors are only connected to $0 \mathrm{~V}$. Moreover, the power consumption in source memristors during VL operation is negligible as shown in (10), therefore the increase of $S_{0}$ only slightly affects $\mathrm{P}_{\mathrm{VL}}$. However, the power consumption in source memristors and in load resistor $\mathrm{R}_{\mathrm{G}}$ during SL operation can significantly increase the $\frac{\mathrm{P}_{\mathrm{SL}}}{\mathrm{P}_{\mathrm{VL}}}$ ratio. When $S_{0}=T$, during SL operation $\left|\mathrm{V}^{+}-\widehat{\mathrm{V}}_{\mathrm{HL}}\right|=\left|\mathrm{V}^{-}-\widehat{\mathrm{V}}_{\mathrm{HL}}\right|$, and thus $\widehat{\mathrm{V}}_{\mathrm{HL}}=0 \mathrm{~V}$ and $\mathrm{P}_{\mathrm{RG}}=0 \mathrm{~V}$. In other words, $\mathrm{P}_{\mathrm{T}}^{S \mathrm{~L}}=\mathrm{P}_{\mathrm{S} 0}^{\mathrm{SL}}$. In this case, $\frac{\mathrm{P}_{\mathrm{SL}}}{\mathrm{P}_{\mathrm{VL}}} \approx 2$. For example, in a $1 \times 8$ crosspoint array for $\left(\mathrm{S}_{0}, \mathrm{~T}\right)=(4,4), \frac{\mathrm{P}_{\mathrm{SL}}}{\mathrm{P}_{\mathrm{VL}}}=2.113$ or in a $1 \times 64$ crosspoint array for $\left(\mathrm{S}_{0}, \mathrm{~T}\right)=(32,32), \frac{\mathrm{P}_{\mathrm{SL}}}{\mathrm{P}_{\mathrm{VL}}}=2.005$.

In a $1 \times 8$ crosspoint array, for any composition of $S_{0}$ and $T, \frac{\mathrm{P}_{\mathrm{SL}}}{\mathrm{PVL}_{\mathrm{VL}}}>1$, i.e. the lower bound of power ratio $\frac{\mathrm{P}_{\mathrm{SL}}}{\mathrm{P}_{\mathrm{VL}}}$ is 1.025 , computed for $\left(S_{0}, T\right)=(1,7)$. This composition maximizes $\mathrm{P}_{\mathrm{VL}}$, while minimizes $\mathrm{P}_{\mathrm{SL}}$ as shown in TABLE 7. However, in a $1 \times n$ crosspoint array where $n>8$, the decrease of $k$ in $\left(S_{0}, T\right)=(k, n-k)$ can bring $\frac{\mathrm{P}_{\mathrm{SL}}}{\mathrm{P}_{\mathrm{VL}}}$ to less than 1, as shown in Table 8. Table 8 shows various compositions of $S_{0}$ and $T$ for which $\frac{\mathrm{P}_{\mathrm{SL}}}{\mathrm{P}_{\mathrm{VL}}}<1$. In all compositions, for any given $S_{0}$, only the lower bound of $T$ for which $\frac{\mathrm{P}_{\mathrm{SL}}}{\mathrm{P}_{\mathrm{VL}}}<1$ is presented, i.e. a larger $T$ leads to a smaller $\frac{\mathrm{P}_{\mathrm{SL}}}{\mathrm{P}_{\mathrm{VL}}}$.

Table 6 Comparison of power ratio $\frac{\mathrm{P}_{\mathrm{SL}}}{\mathrm{P}_{\mathrm{VL}}}$ of a multi-input nor realized in a $1 \times 8$ cross point array with $S_{1}=0$ and various values of $S_{0}$

\begin{tabular}{|c|c|c|c|c|c|c|}
\hline$\left(S_{1}, S_{0}, T\right)$ & Op. & $\begin{array}{c}\mathrm{P}_{\mathrm{S} 0} \\
(\mathrm{nW})\end{array}$ & $\begin{array}{c}\mathrm{P}_{\mathrm{T}} \\
(\mathrm{nW})\end{array}$ & $\begin{array}{c}\mathrm{P}_{\mathrm{RG}} \\
(\mathrm{nW})\end{array}$ & $\begin{array}{c}\mathrm{P}_{\text {avg }} \\
(\mathrm{nW})\end{array}$ & $\begin{array}{c}\mathrm{P}_{\mathrm{SL}} \\
\mathrm{P}_{\mathrm{VL}}\end{array}$ \\
\hline \multirow{2}{*}{$(0,1,1)$} & $\mathrm{VL}$ & $0.556 \mathrm{e}-3$ & 0.556 & 0 & 0.557 & \multirow{2}{*}{2.004} \\
\cline { 2 - 6 }$(0,2,1)$ & $\mathrm{SL}$ & 0.558 & 0.558 & 0 & 1.116 & \\
\hline & $\mathrm{VL}$ & $0.139 \mathrm{e}-3$ & 0.557 & 0 & 0.557 & \multirow{2}{*}{2.975} \\
\cline { 2 - 6 }$(0,4,1)$ & $\mathrm{SL}$ & 0.527 & 0.589 & 0.014 & 1.657 & \\
\cline { 2 - 6 } & $\mathrm{VL}$ & $0.035 \mathrm{e}-3$ & 0.557 & 0 & 0.557 & \multirow{2}{*}{4.772} \\
\cline { 2 - 6 }$(0,7,1)$ & $\mathrm{SL}$ & 0.474 & 0.648 & 0.114 & 2.658 & \\
\cline { 2 - 6 } & $\mathrm{VL}$ & $0.011 \mathrm{e}-3$ & 0.557 & 0 & 0.557 & \multirow{2}{*}{6.427} \\
& $\mathrm{SL}$ & 0.407 & 0.731 & 0.392 & 3.58 & \\
\hline
\end{tabular}


Table 7 Comparison of power ratio $\frac{\mathrm{P}_{\mathrm{SL}}}{\mathrm{P}_{\mathrm{VL}}}$ of a multi-input multi-output nor realized in a $1 \times 8$ cross point array with $S_{1}=0$ and various values of $S_{0}$ and $T$

\begin{tabular}{|c|c|c|c|c|c|c|}
\hline$\left(S_{1}, S_{0}, T\right)$ & Op & $\begin{array}{c}\mathrm{P}_{\mathrm{S} 0} \\
(\mathrm{nW})\end{array}$ & $\begin{array}{c}\mathrm{P}_{\mathrm{T}} \\
(\mathrm{nW})\end{array}$ & $\begin{array}{c}\mathrm{P}_{\mathrm{RG}} \\
(\mathrm{nW})\end{array}$ & $\begin{array}{c}\mathrm{P}_{\text {avg }} \\
(\mathrm{nW})\end{array}$ & $\frac{\mathrm{P}_{\mathrm{SL}}}{\mathrm{P}_{\mathrm{VL}}}$ \\
\hline \multirow[t]{2}{*}{$(0,1,7)$} & $\mathrm{VL}$ & 0.027 & 0.550 & 0 & 3.877 & \multirow[t]{2}{*}{1.025} \\
\hline & SL & 0.731 & 0.407 & 0.392 & 3.972 & \\
\hline \multirow[t]{2}{*}{$(0,2,6)$} & $\mathrm{VL}$ & 0.005 & 0.554 & 0 & 3.352 & \multirow[t]{2}{*}{1.267} \\
\hline & SL & 0.671 & 0.455 & 0.174 & 4.246 & \\
\hline \multirow[t]{2}{*}{$(0,3,5)$} & VL & 0.002 & 0.556 & 0 & 2.786 & \multirow[t]{2}{*}{1.582} \\
\hline & SL & 0.613 & 0.505 & 0.044 & 4.408 & \\
\hline \multirow[t]{2}{*}{$(0,4,4)$} & VL & $0.556 \mathrm{e}-3$ & 0.556 & 0 & 2.226 & \multirow[t]{2}{*}{2.113} \\
\hline & SL & 0.588 & 0.558 & 0 & 4.704 & \\
\hline \multirow[t]{2}{*}{$(0,5,3)$} & $\mathrm{VL}$ & $0.200 \mathrm{e}-3$ & 0.557 & 0 & 1.672 & \multirow[t]{2}{*}{2.636} \\
\hline & SL & 0.505 & 0.613 & 0.044 & 4.408 & \\
\hline \multirow[t]{2}{*}{$(0,6,2)$} & VL & $0.062 \mathrm{e}-3$ & 0.557 & 0 & 1.114 & \multirow[t]{2}{*}{3.811} \\
\hline & SL & 0.455 & 0.671 & 0.174 & 4.246 & \\
\hline \multirow[t]{2}{*}{$(0,7,1)$} & VL & $0.011 \mathrm{e}-3$ & 0.557 & 0 & 0.557 & \multirow[t]{2}{*}{6.427} \\
\hline & SL & 0.407 & 0.731 & 0.392 & 3.972 & \\
\hline
\end{tabular}

Table 8 A multi-input multi-output nor with power ratio $\frac{\mathrm{p}_{\mathrm{sl}}}{\mathrm{P}_{\mathrm{vl}}}<1$ for some combinations of $S_{0}$ and $T$ with $S_{1}=0$ realized in a $1 \times n$ cross point array

\begin{tabular}{|c|c|c|c|c|c|c|}
\hline$\left(S_{1}, S_{0}, T\right)$ & Op & $\begin{array}{c}\mathrm{P}_{\mathrm{S} 0} \\
\text { (nW) }\end{array}$ & $\begin{array}{c}\mathrm{P}_{\mathrm{T}} \\
(\mathrm{nW})\end{array}$ & $\begin{array}{c}\mathrm{P}_{\mathrm{RG}} \\
(\mathrm{nW})\end{array}$ & $\begin{array}{c}\mathrm{P}_{\text {avg }} \\
(\mathrm{nW})\end{array}$ & $\frac{\mathrm{P}_{\mathrm{SL}}}{\mathrm{P}_{\mathrm{VL}}}$ \\
\hline \multirow[t]{2}{*}{$(0,1,8)$} & VL & 0.035 & 0.549 & 0 & 4.427 & \multirow[t]{2}{*}{0.987} \\
\hline & SL & 0.757 & 0.388 & 0.508 & 4.369 & \\
\hline \multirow[t]{2}{*}{$(0,2,12)$} & VL & 0.020 & 0.551 & 0 & 6.652 & \multirow[t]{2}{*}{0.997} \\
\hline & SL & 0.818 & 0.347 & 0.830 & 6.63 & \\
\hline \multirow[t]{2}{*}{$(0,3,16)$} & VL & 0.016 & 0.552 & 0 & 8.88 & \multirow[t]{2}{*}{0.990} \\
\hline & SL & 0.869 & 0.315 & 1.147 & 8.794 & \\
\hline \multirow[t]{2}{*}{$(0,4,20)$} & VL & 0.014 & 0.552 & 0 & 11.096 & \multirow[t]{2}{*}{0.982} \\
\hline & SL & 0.912 & 0.290 & 1.447 & 10.895 & \\
\hline \multirow[t]{2}{*}{$(0,5,23)$} & VL & 0.012 & 0.552 & 0 & 12.756 & \multirow[t]{2}{*}{0.992} \\
\hline & SL & 0.933 & 0.278 & 1.601 & 12.66 & \\
\hline \multirow[t]{2}{*}{$(0,6,26)$} & VL & 0.010 & 0.553 & 0 & 14.438 & \multirow[t]{2}{*}{0.998} \\
\hline & SL & 0.951 & 0.268 & 1.742 & 14.416 & \\
\hline \multirow[t]{2}{*}{$(0,7,30)$} & VL & 0.010 & 0.553 & 0 & 16.66 & \multirow[t]{2}{*}{0.987} \\
\hline & SL & 0.982 & 0.253 & 1.987 & 16.451 & \\
\hline
\end{tabular}

\subsection{Analysis of power consumption and propagation delays in a $1 \times 64$ crosspoint arrays for $S_{1}>0$}

Tables 9, 10, 11 and 12 compare the switching delay and the overall power consumption in a $1 \times 64$ crosspoint array during SL and VL for various compositions of $S_{1}, S_{0}$, and $T$, where $S_{1}>0$. All simulation results, shown in Tables 9 through 12, are consistent with the first four observations stated for a $1 \times 8$ crosspoint array in Section 5.1. However, unlike the last two observations stated for a $1 \times 8$ crosspoint array, the following is observed.

1) The main contributors to the power ratio $\frac{\mathrm{P}_{\mathrm{SL}}}{\mathrm{P}_{\mathrm{VL}}}$ are $S_{0}, T$ and $\mathrm{P}_{\mathrm{RG}}$. For example, holding $T$ constant while increasing $S_{0}$, as shown in Table 9, decreases the power ratio $\frac{\mathrm{P}_{\mathrm{SL}}}{\mathrm{P}_{\mathrm{VL}}}$. In contrast, holding $S_{0}$ constant while increasing $T$, as shown in Table 10 , brings $\frac{\mathrm{P}_{\mathrm{SL}}}{\mathrm{P}_{\mathrm{VL}}}$ close to 1 . 
2) The propagation delay $t_{d}$ in VL is shorter than in $\mathrm{SL}$ if $\frac{\mathrm{P}_{\mathrm{S} 1}^{\mathrm{SL}}}{\mathrm{P}_{\mathrm{S} 1}^{\mathrm{VL}}}>1$, and is equal or longer than in $\mathrm{SL}$ if $\frac{\mathrm{P}_{\mathrm{SL}}^{\mathrm{SL}}}{\mathrm{P}_{\mathrm{S} 1}^{\mathrm{VL}}} \leq 1$.

In a $1 \times 64$ crosspoint array, for any composition of $S_{1}, S_{0}$, and $T$, where $S_{1}>0$, equations (5), (7) and (8) hold, and since $7.078 \leq \frac{\mathrm{P}_{\mathrm{RG}}}{\mathrm{P}_{\mathrm{T}}^{S L}} \leq 8.328$, equation (6) can still approximate the relation between $\mathrm{P}_{\mathrm{RG}}$ and $\mathrm{P}_{\mathrm{T}}^{\mathrm{SL}}$ during $\mathrm{SL}$ operation. As a result, power ratio $\frac{\mathrm{P}_{\mathrm{SL}}}{\mathrm{P}_{\mathrm{VL}}}$ can still be approximated as in (9). All derivations are in the same manner as shown in Section 5.1.

Table 9 shows that VL consumes more power than SL when $S_{0}>33$; in this case, an increase of $T$ brings the $\frac{\mathrm{P}_{\mathrm{SL}}}{\mathrm{P}_{\mathrm{VL}}}$ ratio close to 1 as predicted by (9). This prediction is confirmed in Table 10. The converse is also true, when $S_{0}<33$, VL consumes less power than SL and the increase of $T$ brings the $\frac{\mathrm{PSL}_{\mathrm{SL}}}{\mathrm{P}_{\mathrm{VL}}}$ ratio close to 1 , as shown in Table 11. Note that the increase of $S_{1}$ slightly affects the $\frac{\mathrm{P}_{\mathrm{SL}}}{\mathrm{P}_{\mathrm{VL}}}$ ratio as shown in Table 12.

In terms of propagation delay, volistor gates perform faster than stateful gates when $\frac{\mathrm{P}_{\mathrm{S1}}^{\mathrm{SL}}}{\mathrm{P}_{\mathrm{S} 1}^{\mathrm{VL}}}>1$. When $\frac{\mathrm{P}_{\mathrm{S1}}^{\mathrm{SL}}}{\mathrm{P}_{\mathrm{S} 1}^{\mathrm{VL}}} \approx 1$, the propagation delay in both operations is almost the same; when $\frac{\mathrm{P}_{\mathrm{S1}}^{\mathrm{SL}}}{\mathrm{P}_{\mathrm{S} 1}^{\mathrm{VL}}}<1$, stateful gates perform faster than volistors. In other words, the propagation delay changes with the voltage on the common nanowire of a crosspoint array or equivalently the voltage drop across the target memristors. A logic gate performs faster if the voltage drop across its target memristors is larger. The increase of $S_{0}$ versus the decrease of $S_{1}$ results in faster stateful gates and vice versa as shown in Table 9. In addition, the increase of $\mathrm{T}$ versus the decrease of $\mathrm{S}_{1}$ results in faster volistor gates. Table 11 shows the increase of $t_{d}$ in SL versus VL for constant $S_{0}$ with T varied from 1 to 57.

Table 9 Comparison of delay $t_{d}$ and power ratio $\frac{p_{\mathrm{sl}}}{p_{\mathrm{vl}}}$ of a multi-input nor realized in a $1 \times 64$ cross point array with $S_{0} \geq 33$ and varied $S_{1}$

\begin{tabular}{|c|c|c|c|c|c|c|c|c|}
\hline$\left(S_{1}, S_{0}, T\right)$ & Op. & $\begin{array}{c}\mathrm{P}_{\mathrm{S} 0} \\
(\mathrm{nW})\end{array}$ & $\begin{array}{c}\mathrm{P}_{\mathrm{S} 0} \\
(\mathrm{nW})\end{array}$ & $\begin{array}{c}\mathrm{P}_{\mathrm{T}} \\
(\mathrm{nW})\end{array}$ & $\begin{array}{c}\mathrm{P}_{\mathrm{RG}} \\
(\mathrm{nW})\end{array}$ & $\begin{array}{c}\mathrm{t}_{\mathrm{d}} \\
(\mathrm{ns})\end{array}$ & $\begin{array}{c}\mathrm{P}_{\text {avg }} \\
(\mathrm{nW})\end{array}$ & $\frac{\mathrm{P}_{\mathrm{SL}}}{\mathrm{P}_{\mathrm{VL}}}$ \\
\hline \multirow[t]{2}{*}{$(30,33,1)$} & VL & $0.757 \mathrm{e}-3$ & 0.556 & 2.228 & 0 & 4.041 & 20.599 & \multirow[t]{2}{*}{1.009} \\
\hline & SL & $0.770 \mathrm{e}-3$ & $0.770 \mathrm{e}-6$ & 2.228 & 18.541 & 4.041 & 20.792 & \\
\hline \multirow[t]{2}{*}{$(29,34,1)$} & VL & $0.857 \mathrm{e}-3$ & 0.556 & 2.227 & 0 & 4.034 & 21.156 & \multirow[t]{2}{*}{0.982} \\
\hline & SL & $0.824 \mathrm{e}-3$ & $0.824 \mathrm{e}-6$ & 2.227 & 18.539 & 4.042 & 20.767 & \\
\hline \multirow[t]{2}{*}{$(25,38,1)$} & VL & $1.423 \mathrm{e}-3$ & 0.558 & 2.227 & 0 & 4.048 & 23.467 & \multirow[t]{2}{*}{0.885} \\
\hline & SL & $1.107 \mathrm{e}-3$ & $1.107 \mathrm{e}-6$ & 2.227 & 18.533 & 4.044 & 20.760 & \\
\hline \multirow[t]{2}{*}{$(13,44,1)$} & VL & 0.007 & 0.554 & 2.222 & 0 & 4.072 & 26.689 & \multirow[t]{2}{*}{0.778} \\
\hline & SL & 0.004 & $4.069 \mathrm{e}-6$ & 2.224 & 18.484 & 4.060 & 20.760 & \\
\hline \multirow[t]{2}{*}{$(1,62,1)$} & $\mathrm{VL}$ & 2.021 & 0.492 & 2.098 & 0 & 4.907 & 34.623 & \multirow[t]{2}{*}{0.583} \\
\hline & SL & 0.579 & $0.579 \mathrm{e}-3$ & 2.159 & 17.406 & 4.470 & 20.18 & \\
\hline
\end{tabular}


Table 9 shows $\frac{\mathrm{P}_{\mathrm{SL}}}{\mathrm{P}_{\mathrm{VL}}}<1$ when $S_{0}>33$, regardless of the $S_{1}$ value. Table 12 reinforces our conclusion that $S_{1}$ has almost no effect on the power ratio, $\frac{\mathrm{P}_{\mathrm{SL}}}{\mathrm{P}_{\mathrm{VL}}}$. The increase of $S_{0}$, and the decrease of $S_{1}$ result in $\mathrm{P}_{\mathrm{S} 1}^{\mathrm{VL}}>\mathrm{P}_{\mathrm{S} 1}^{\mathrm{SL}}$ and thus a longer switching delay $t_{d}$ for VL.

Table 10 Comparison of delay $t_{d}$ and power ratio $\frac{p_{\mathrm{s} 1}}{p_{\mathrm{vl}}}$ of a multi-input nor realized in a $1 \times 64$ cross point array with constant input values $S_{0}$ and $S_{1}$, and varied $T$

\begin{tabular}{|c|c|c|c|c|c|c|c|c|}
\hline$\left(S_{1}, S_{0}, T\right)$ & Op. & $\begin{array}{l}\mathrm{P}_{\mathrm{S} 1} \\
(\mathrm{nW})\end{array}$ & $\begin{array}{l}\mathrm{P}_{\mathrm{S} 0} \\
(\mathrm{nW})\end{array}$ & $\begin{array}{l}\mathrm{P}_{\mathrm{T}} \\
(\mathrm{nW})\end{array}$ & $\begin{array}{l}\mathrm{P}_{\mathrm{RG}} \\
(\mathrm{nW})\end{array}$ & $\begin{array}{l}\mathrm{t}_{\mathrm{d}} \\
\text { (ns) }\end{array}$ & $\begin{array}{l}\mathrm{P}_{\text {avg }} \\
(\mathrm{nW})\end{array}$ & $\frac{\mathrm{P}_{\mathrm{SL}}}{\mathrm{P}_{\mathrm{VL}}}$ \\
\hline \multirow[t]{2}{*}{$(1,40,1)$} & VL & 0.908 & 0.513 & 2.141 & 0 & 4.575 & 23.569 & \multirow[t]{2}{*}{0.856} \\
\hline & SL & 0.603 & $0.603 e-3$ & 2.157 & 17.383 & 4.461 & 20.167 & \\
\hline \multirow[t]{2}{*}{$(1,40,4)$} & VL & 1.179 & 0.507 & 2.129 & 0 & 4.665 & 29.975 & \multirow[t]{2}{*}{0.888} \\
\hline & SL & 0.821 & $0.821 \mathrm{e}-3$ & 2.145 & 17.187 & 4.543 & 26.621 & \\
\hline \multirow[t]{2}{*}{$(1,40,8)$} & VL & 1.592 & 0.5 & 2.113 & 0 & 4.780 & 38.496 & \multirow[t]{2}{*}{0.914} \\
\hline & SL & 1.161 & $1.161 \mathrm{e}-3$ & 2.130 & 16.928 & 4.65 & 35.175 & \\
\hline \multirow[t]{2}{*}{$(1,40,16)$} & VL & 2.592 & 0.484 & 2.081 & 0 & 5.049 & 55.248 & \multirow[t]{2}{*}{0.943} \\
\hline & SL & 2.005 & $2.005 \mathrm{e}-3$ & 2.098 & 16.421 & 4.895 & 52.074 & \\
\hline
\end{tabular}

Table 10 shows how the increase of $T$ affects $\frac{\mathrm{P}_{\mathrm{SL}}}{\mathrm{P}_{\mathrm{VL}}}$ when $S_{0}>33$. The increase of $T$ brings $\frac{\mathrm{P}_{\mathrm{SL}}}{\mathrm{P}_{\mathrm{VL}}}$ close to 1 . For all compositions, $\mathrm{t}_{\mathrm{d}}$ is longer for $\mathrm{VL}$. This long propagation delay is related to the increase of $T$ and the large $S_{0}$.

Table 11 Comparison of delay $t_{d}$ and power ratio $\frac{p_{s 1}}{p_{v 1}}$ of a multi-input nor realized in a $1 \times 64$ cross point array with constant number of inputs and varied outputs

\begin{tabular}{|c|c|c|c|c|c|c|c|c|}
\hline$\left(S_{1}, S_{0}, T\right)$ & Op. & $\begin{array}{l}\mathrm{P}_{\mathrm{S} 1} \\
(\mathrm{nW})\end{array}$ & $\begin{array}{c}\mathrm{P}_{\mathrm{S} 0} \\
(\mathrm{nW})\end{array}$ & $\begin{array}{c}\mathrm{P}_{\mathrm{T}} \\
(\mathrm{nW})\end{array}$ & $\begin{array}{c}\mathrm{P}_{\mathrm{RG}} \\
(\mathrm{nW})\end{array}$ & $\begin{array}{c}\mathrm{t}_{\mathrm{d}} \\
(\mathrm{ns})\end{array}$ & $\begin{array}{l}\mathrm{P}_{\text {avg }} \\
(\mathrm{nW})\end{array}$ & $\frac{\mathrm{P}_{\mathrm{SL}}}{\mathrm{P}_{\mathrm{VL}}}$ \\
\hline \multirow[t]{2}{*}{$(1,6,1)$} & $\mathrm{VL}$ & 0.035 & 0.549 & 2.212 & 0 & 4.103 & 5.541 & \multirow[t]{2}{*}{3.636} \\
\hline & SL & 0.643 & $0.643 \mathrm{e}-3$ & 2.155 & 17.345 & 4.454 & 20.147 & \\
\hline \multirow[t]{2}{*}{$(1,6,5)$} & VL & 0.140 & 0.54 & 2.195 & 0 & 4.223 & 14.355 & \multirow[t]{2}{*}{2.002} \\
\hline & SL & 0.960 & $0.96 \mathrm{e}-3$ & 2.139 & 17.074 & 4.571 & 28.735 & \\
\hline \multirow[t]{2}{*}{$(1,6,15)$} & VL & 0.693 & 0.519 & 2.152 & 0 & 4.474 & 36.087 & \multirow[t]{2}{*}{1.383} \\
\hline & SL & 2.020 & $2.020 \mathrm{e}-3$ & 2.098 & 16.420 & 4.874 & 49.922 & \\
\hline \multirow[t]{2}{*}{$(1,6,30)$} & VL & 2.263 & 0.489 & 2.090 & 0 & 4.942 & 67.897 & \multirow[t]{2}{*}{1.192} \\
\hline & SL & 4.247 & $4.247 \mathrm{e}-3$ & 2.040 & 15.482 & 5.411 & 80.954 & \\
\hline \multirow[t]{2}{*}{$(1,6,57)$} & VL & 7.104 & 0.439 & 1.985 & 0 & 6.026 & 122.883 & \multirow[t]{2}{*}{1.096} \\
\hline & SL & 10.069 & $10.069 \mathrm{e}-3$ & 1.94 & 13.925 & 6.681 & 134.634 & \\
\hline
\end{tabular}

Table 11 shows how the increase of $T$ affects $\frac{\mathrm{P}_{\mathrm{SL}}}{\mathrm{P}_{\mathrm{VL}}}$ when $S_{1}$ and $S_{0}$ are kept constant at 1 and 6, respectively. For all compositions, shown in Table 11, $\frac{\mathrm{P}_{\mathrm{RG}}}{S_{0} \times \mathrm{P}_{\mathrm{SL}}^{\mathrm{VL}}} \approx 5.2$; however the increase of $T$ brings $\frac{\mathrm{P}_{\mathrm{SL}}}{\mathrm{P}_{\mathrm{VL}}}$ close to 1, reducing the effect of $\frac{\mathrm{P}_{\mathrm{RG}}}{S_{0} \times \mathrm{P}_{\mathrm{S} 0}^{\mathrm{VL}}}$ in $\frac{\mathrm{P}_{\mathrm{SL}}}{\mathrm{P}_{\mathrm{VL}}}$ significantly as predicted by (9). As a result, executing a multi-input multi-output gate in a small crosspoint array, e.g. $1 \times 8$, with VL is more power efficient than with SL. This conclusion is consistent with (9) and with the simulation results shown in Table 5. The increase of $T$ results in $\mathrm{P}_{\mathrm{S} 1}^{\mathrm{SL}}>\mathrm{P}_{\mathrm{S} 1}^{\mathrm{VL}}$ and thus longer $\mathrm{t}_{\mathrm{d}}$ for SL. 
Table 12 Comparison of delays $t_{d}$ and power ratio $\frac{p_{\mathrm{sl}}}{p_{\mathrm{vl}}}$ of a multi-input nor realized in a $1 \times 64$ cross point array with varying $S_{1}$ and $S_{0}>33$

\begin{tabular}{|c|l|l|l|l|l|l|l|c|}
\hline$\left(S_{1}, S_{0}, T\right)$ & Op. & $\begin{array}{l}\mathrm{P}_{\mathrm{S} 1} \\
(\mathrm{nW})\end{array}$ & $\begin{array}{l}\mathrm{P}_{\mathrm{S} 0} \\
(\mathrm{nW})\end{array}$ & $\begin{array}{l}\mathrm{P}_{\mathrm{T}} \\
(\mathrm{nW})\end{array}$ & $\begin{array}{l}\mathrm{P}_{\mathrm{RG}} \\
(\mathrm{nW})\end{array}$ & $\begin{array}{l}\mathrm{t}_{\mathrm{d}} \\
(\mathrm{ns})\end{array}$ & $\begin{array}{l}\mathrm{P}_{\text {avg }} \\
(\mathrm{nW})\end{array}$ & $\frac{\mathrm{P}_{\mathrm{SL}}}{\mathrm{P}_{\mathrm{VL}}}$ \\
\hline \multirow{2}{*}{$(1,40,1)$} & $\mathrm{VL}$ & 0.908 & 0.513 & 2.141 & 0 & 4.585 & 23.569 & 0.856 \\
\cline { 2 - 9 } & $\mathrm{SL}$ & 0.603 & $0.603 \mathrm{e}-3$ & 2.157 & 17.383 & 4.468 & 20.167 & \\
\hline \multirow{2}{*}{$(5,40,1)$} & $\mathrm{VL}$ & 0.039 & 0.548 & 2.212 & 0 & 4.140 & 24.327 & 0.850 \\
\cline { 2 - 8 } & $\mathrm{SL}$ & 0.027 & $0.027 \mathrm{e}-3$ & 2.215 & 18.326 & 4.122 & 20.677 & \\
\hline \multirow{2}{*}{$(10,40,1)$} & $\mathrm{VL}$ & 0.010 & 0.553 & 2.221 & 0 & 4.079 & 24.441 & \multirow{2}{*}{0.849} \\
\cline { 2 - 8 } & SL & 0.007 & $0.007 \mathrm{e}-3$ & 2.223 & 18.456 & 4.080 & 20.749 & \\
\hline \multirow{2}{*}{$(20,40,1)$} & $\mathrm{VL}$ & 0.002 & 0.555 & 2.226 & 0 & 4.064 & 24.466 & \multirow{2}{*}{0.849} \\
\cline { 2 - 7 } & SL & $1.727 \mathrm{e}-3$ & $1.727 \mathrm{e}-6$ & 2.226 & 18.52 & 4.057 & 20.781 & \\
\hline
\end{tabular}

Table 12 shows how the increase of $S_{1}$ affects $\frac{\mathrm{P}_{\mathrm{SL}}}{\mathrm{PVL}_{\mathrm{VL}}}$ when $S_{0}>33$ and $T$ are kept constant at 40 and 1, respectively.

The increase of $S_{1}$ has almost no effect on $\frac{\mathrm{P}_{\mathrm{SL}}}{\mathrm{P}_{\mathrm{VL}}}$, i.e. all compositions result in almost the same power ratio 0.856 . Although the increase of $S_{1}$ has almost no effect on $\frac{\mathrm{P}_{\mathrm{SL}}}{\mathrm{P}_{\mathrm{VL}}}$, it does decrease the propagation delay in both SL and VL operations.

As a summary, in a $1 \times 8$ crosspoint array, the power consumption in SL is higher than in VL and volistors operate faster than stateful gates. In a $1 \times 64$ crosspoint array, when $S_{1}>0$, the majority of the power consumption in VL is $S_{0} \times \mathrm{P}_{\mathrm{S} 0}^{\mathrm{VL}}+T \times \mathrm{P}_{\mathrm{T}}$, and in $\mathrm{SL}$ is $\mathrm{P}_{\mathrm{RG}}+T \times \mathrm{P}_{\mathrm{T}}$ as described in (5)-(8). In this case, power ratio $\frac{\mathrm{P}_{\mathrm{SL}}}{\mathrm{P}_{\mathrm{VL}}}$ can be approximated with (9). In a $1 \times 64$ crosspoint array, a multi-input volistor gate consumes less power than a multi-input stateful gate, unless the majority of inputs are logic ' 0 '. When $S_{1}=0$, a multi-input volistor gate consumes less power than a multi-input stateful gate. In a $1 \times 64$ crosspoint array, for $S_{1}>0$, a logic gate with high fan-out consumes the same power, regardless of the implementation, SL or VL. When $S_{1}=0$, the majority of the power consumption in VL is $T \times \mathrm{P}_{\mathrm{T}}^{\mathrm{VL}}$ as shown in (10). However, in SL operation, the power consumption in each circuit element depends on the combination of $S_{0}$ and $T$.

\section{MEMORY APPLICATION}

Memristors are nanoscale nonvolatile devices used primarily as resistive memory, e.g. resistance RAM. The generic structure for memristive memory is a crossbar array. The crossbar arrays can be vertically stacked creating a 3D crossbar memory [16]. The potential problem of the crossbar structure is the leakage pathways from surrounding memory cells. In a recent work [17], Vourkas et al proposed memories comprised of parallel/serial complementary 
memristive switches. In order to have states that are distinguishable, they introduced insulating junctions within a crossbar array to restrain leakage pathways [18].

In another approach, Kim et al fabricated a crossbar array utilizing rectifying memristors rather than standard memristors [4]. The use of rectifying memristors eliminates the leakage pathways due to the diode like behavior of

memristors, i.e. the small $\frac{I^{-}}{I^{+}}$ratio, see Section 2. Furthermore, the large $\frac{R_{O P E N}}{R_{C L O S E D}}$ ratio of the rectifying memristors allows for multilevel memory as demonstrated in [4]. This memory array can be programmed exploiting volistor gates. As demonstrated in Section 5, volistor gates are more efficient than stateful gates in terms of power consumption. In the process of programming the memory array, first, all memristors are initialized to low resistance state. This initialization step can be implemented in one clock cycle. Second, every row or column of memristors from the memory array can be programmed within one clock cycle. This step requires $m$ or $n$ clock cycles to program all memory cells of an $m \times n$ memory array. The latter approach for realization of memory is more efficient than the former approach in terms of simplicity, power consumption, reliability, and robustness e.g. the second approach allows for realization of multilevel memory. Furthermore, realization of memory array with rectifying memristors allows for programming the memory cells exploiting volistors, which is a more power-efficient approach than the conventional stateful approach. This distinction between memories is originated from the property of rectifying memristors over standard memristors.

Volistors can be used to create memory-like digital sensor integrating devices that read voltages from many sensors in parallel/serial mode and convert them to words of a stored resistive information that can be next processed by combinational stateful memristive circuits.

\section{CONCLUSION}

Volistors capitalize on the characteristics of rectifying memristors to allow voltages to be used directly as inputs. There is no need to store inputs as resistances in memristors before logic can be performed. This eliminates the use of $\mathrm{R}_{\mathrm{G}}$ to read out the inputs in the first logic level and thus eliminates the most power-hungry circuit element in stateful logic. There is no static power in our circuits. The switching power consumed by volistor logic in a crosspoint array is entirely due to the leakage through the reverse biased rectifying memristor as there is no direct path to the ground. Using volistors at the first level provides NOR and AND directly which provides a flexibility sufficient to remove the 
need for IMP gates in stateful logic and to realize all subsequent levels with only NOR/NOT. Realizing all stateful logic levels with NOR/NOT simplifies the driver circuitry and removes the need for keeper circuits. An additional advantage is that the first-level volistor logic can have as many inputs as the number of available memristors in the crosspoint array within a single operation. However, inputs set to $0 \mathrm{~V}$ incur a higher power penalty than the inputs set to $\mathrm{V}^{+}$or $\mathrm{V}^{-}$. The two-dimensional, multi-output property of volistors provides for both a large fan-out (at the cost of increased power consumption) and for a great flexibility in the placement of calculation results. Using multiple crossbars in a network provides parallelization leading to pipelined and SIMD architectures in the data path. Several different first-level volistor operations can be computed in separate crossbars at the same time, greatly speeding up what is typically the most complex level of implementing Boolean functions. Subsequent logic levels can be accomplished in connected crossbars in an approach that uses mixed-input gates and stateful NOR. This hybrid approach with volistors and stateful NOR can be used to compute logic faster, with less power and with simpler external control circuitry than a solely stateful approach.

\section{ACKNOWLEDGMENT}

This work was supported by the Higher Committee for Education Development in Iraq (HCED).

\section{REFERENCES}

[1] Borghetti J, Snider G, Kuekes P, Yang J, Stewart D, Williams R (2010) Memristive' switches enable 'stateful' logic operations via material implication. Nature 464(7290): 873-876.

[2] Lehtonen E, Poikonen J. H, Laiho M (2012) Applications and limitations of memristive implication logic. In 2012 13th International Workshop on Cellular Nanoscale Networks and Their Applications (CNNA): 1-6.

[3] Kim K, Shin S, Kang S (2011) Field programmable stateful logic array. IEEE Trans Comput-Aided Design Integr Circuits Syst 30(12): 18001813.

[4] Kim K, Gaba S, Wheeler D, Cruz-Albrecht J, Hussain T, Srinivasa N, Lu W (2012) A Functional Hybrid Memristor Crossbar-Array/CMOS System for Data Storage and Neuromorphic Applications. Nano Letters 12(1): 389-395.

[5] Kvatinsky S, Wald N, Satat G, Kolodny A, Weiser U.C, Friedman E.G (2012) MRL-memristor ratioed logic. In 2012 13th International Workshop on Cellular Nanoscale Networks and Their Applications (CNNA): 1-6.

[6] Kim K.H, Hyun Jo S, Gaba S, Lu W (2010) Nanoscale resistive memory with intrinsic diode characteristics and long endurance. Appl Phys Lett 96(5): 053106.

[7] Lehtonen E, Tissari J, Poikonen J, Laiho M, Koskinen L (2014) A cellular computing architecture for parallel memristive stateful logic. Microelectronics Journal 45(11): 1438-1449.

[8] Lee M J, Lee C B, Lee D, Lee S R, Chang M, Hur JH, Kim Y B, Kim C J, Seo D H, Seo S, Chung U I (2011) A fast, high-endurance and scalable non-volatile memory device made from asymmetric Ta2O5- $\mathrm{x} / \mathrm{TaO} 2-\mathrm{x}$ bilayer structures. Nature materials 10(8): 625-630.

[9] Torrezan A C, Strachan J P, Medeiros-Ribeiro G, Williams R S (2011) Sub-nanosecond switching of a tantalum oxide memristor. Nanotechnology 22(48): 485203.

[10] Lehtonen E, Poikonen J, Laiho M (2010) Two memristors suffice to compute all Boolean functions. Electronics letters 46(3): 230.

[11] Raghuvanshi A, Perkowski M (2014) Logic synthesis and a generalized notation for memristor-realized material implication gates. In Proceedings of the 2014 IEEE/ACM International Conference on Computer-Aided Design: 470-477.

[12] Gimpel J. F (1967) The Minimization of TANT Networks. IEEE Transactions on Electronic Computers 16(1): 18-38.

[13] Laiho M, Lehtonen E (2010) Cellular nanoscale network cell with memristors for local implication. In Proceedings of the IEEE International Symposium on Circuits and Systems, ISCAS 2010.

[14] Perkowski M, Fiszerl R, Kerntopf P, Lukac M (2012) An Approach to Synthesis of Reversible Circuits for Partially Specified Functions. In: 2012 12th IEEE Conference on Nanotechnology (IEEE-NANO): 1-6.

[15] Jo S, Kim K, Lu W (2009) High-Density Crossbar Arrays Based on a Si Memristive System. Nano Letters 9(2): 870-874.

[16] Strukov D B, Williams R S (2009) Four-dimensional address topology for circuits with stacked multilayer crossbar arrays. Proceedings of the National Academy of Sciences 106:20155-20158.

[17] Vourkas I, Sirakoulis G C (2014) Nano-crossbar memories comprising parallel/serial complementary memristive switches. BioNanoScience 4: 166-179. 
[18] Vourkas I, Stathis D, Sirakoulis G C (2013) Improved read voltage margins with alternative topologies for memristor-based crossbar memories. In VLSI-SoC: 336-339. 